\title{
TOPOLOGICAL EQUIVALENCE OF FLOWS ON HOMOGENEOUS SPACES, AND DIVERGENCE OF ONE-PARAMETER SUBGROUPS OF LIE GROUPS
}

\author{
DIEGO BENARDETE
}

\begin{abstract}
Let $\Gamma$ and $\Gamma^{\prime}$ be lattices, and $\phi$ and $\phi^{\prime}$ one-parameter subgroups of the connected Lie groups $G$ and $G^{\prime}$. If one of the following conditions (a), (b), or (c) hold, Theorem A states that if the induced flows on the homogeneous spaces $G / \Gamma$ and $G^{\prime} / \Gamma^{\prime}$ are topologically equivalent, then they are topologically equivalent by an affine map. (a) $G$ and $G^{\prime}$ are one-connected and nilpotent. (b) $G$ and $G^{\prime}$ are one-connected and solvable, and for all $X$ in $L(G)$ and $X^{\prime}$ in $L\left(G^{\prime}\right)$, ad $(x)$ and $\operatorname{ad}\left(X^{\prime}\right)$ have only real eigenvalues. (c) $G$ and $G^{\prime}$ are centerless and semisimple with no compact direct factor and no direct factor $H$ isomorphic to $\operatorname{PSL}(2, R)$ such that $\Gamma H$ is closed in $G$. Moreover, in condition (c), the induced flow of $\phi$ on $G / \Gamma$ is assumed to be ergodic.

Theorem A depends on Theorem B, which concerns divergence properties of one-parameter subgroups. We say $\phi$ is isolated if and only if for any $\phi^{\prime}$ which recurrently approaches $\phi$ for positive and negative time, $\phi$ equals $\phi^{\prime}$ up to sense-preserving reparameterization. Theorem B(a) states that if $G$ is oneconnected and nilpotent, or one-connected and solvable with exp: $L(G) \rightarrow G$ a diffeomorphism, then every $\phi$ of $G$ is isolated. Let $G$ be connected and semisimple and $\phi(t)=\exp (t X)$. Then Theorem $\mathrm{B}(\mathrm{b})$ states that $\phi$ is isolated, if $[X, Y]=0$ and $\operatorname{ad}(Y)$ being semisimple imply that $\operatorname{ad}(Y)$ has some eigenvalue not pure imaginary and not zero. If $G$ has finite center, $\phi$ is isolated if there is no compact connected subgroup in the centralizer of $\phi$.
\end{abstract}

1. Introduction. Let $G$ be a connected Lie group, $\Gamma$ a discrete subgroup, $\phi: \mathbf{R} \rightarrow G$ a one-parameter subgroup, and $\phi^{*}:(\mathbf{R} \times G / \Gamma) \rightarrow G / \Gamma$, where $\phi^{*}:(t, x \Gamma)$ $\mapsto(\phi(t) x) \Gamma$, the $G$-induced flow. $G / \Gamma$ is a homogeneous space.

Since the book Flows on homogeneous spaces by Auslander Green, and Hahn [3], the ergodic theory and topological dynamics of these flows have been much studied because they provide interesting examples which can be understood in this algebraic setting. (See for example, Auslander [2], Brezin and Moore [5].) The equivalences studied have usually been parameter preserving, measure theoretic or topological maps. (See Parry [23], Walters [31], Ratner [27, 28], and Witte [32].) In this paper we study topological equivalences which do not preserve the parameter. We use results concerning the divergence of one-parameter subgroups. These results may have their own geometric interest.

Received by the editors December 2, 1985 and, in revised form, September 3, 1986.

1980 Mathematics Subject Classification (1985 Revision). Primary 58F25; Secondary 22E40, 22E15.

Key words and phrases. Topological equivalence, flows, homogeneous space, one-parameter subgroups, Lie groups, discrete subgroups, lattice. 
DEFINITION. Two flows are topologically equivalent if and only if there is a homeomorphism taking trajectories to trajectories, preserving the sense but not necessarily the parameterization.

DEFINITION. A topological conjugacy of flows is a parameter preserving topological equivalence.

DEFINITION. Let $G$ and $G^{\prime}$ be Lie groups and $\Gamma$ and $\Gamma^{\prime}$ discrete subgroups. An affine map from $G / \Gamma$ to $G / \Gamma^{\prime}$ is a map of the form $\bar{a} \bar{A}: x \Gamma \mapsto(a(A x)) \Gamma^{\prime}$ where $a \in G^{\prime}$ and $A$ is an isomorphism of $G$ onto $G^{\prime}$ which extends an isomorphism of $\Gamma$ onto $\Gamma^{\prime}$.

DEFINITION. Two flows are affinely equivalent (affinely conjugate) if and only if they are topologically equivalent (topologically conjugate) by an affine map.

EXAMPLE. For $a \in G$, the affine map $\bar{a}: G / \Gamma \rightarrow G / \Gamma$ is an affine conjugacy (and therefore an affine equivalence) of the flows induced by the one-parameter subgroups $\phi$ and $a \phi a^{-1}$ (see $\S 2$ (Lemma 1$)$ ).

DEfinition. A discrete subgroup $\Gamma$ is a lattice of $G$ if and only if there exists a finite Borel measure on $G / \Gamma$ invariant under affine maps of the form $\bar{a}: x \Gamma \mapsto(a x) \Gamma$, for all $a$ in $G$.

DEFINITION. $H$ is a uniform subgroup of $G$ if and only if $G / H$ is compact.

A uniform discrete subgroup is a lattice [26].

Notation. For any Lie group $G, L(G)$ is its Lie algebra.

THEOREM A. Let $G$ and $G^{\prime}$ be connected Lie groups, $\Gamma$ and $\Gamma^{\prime}$ be lattices of $G$ and $G^{\prime}$, and $\phi^{*}$ and $\phi^{* *}$ flows on $G / \Gamma$ and $G^{\prime} / \Gamma^{\prime}$ induced by one-parameter subgroups $\phi$ and $\phi^{\prime}$ of $G$ and $G^{\prime}$. If one of the following conditions (a), (b), or (c) hold, then $\phi^{*}$ and $\phi^{\prime *}$ are topologically equivalent if and only if they are affinely equivalent.

CONDITION (a). $G$ and $G^{\prime}$ are simply connected and nilpotent.

Condition (b). $G$ and $G^{\prime}$ are simply connected and solvable, and, for any $X$ in $L(G)$ and $X^{\prime}$ in $L\left(G^{\prime}\right), \operatorname{ad}(X)$ and $\operatorname{ad}\left(X^{\prime}\right)$ have only real eigenvalues.

CONDITION (c). $G$ and $G^{\prime}$ are semisimple with trivial center and no compact direct factor. There is no direct factor $H$ of $G$, such that both $H$ is isomorphic to $\operatorname{PSL}(2, \mathbf{R})$ and $\Gamma H$ is a closed subgroup of $G$. Furthermore, $\phi$ must satisfy condition c(1) or condition $\mathrm{c}(2)$. $\phi$.

CONDITION $\mathrm{c}(1)$. There is no compact connected subgroup in the centralizer of

CONDITION $\mathrm{c}(2)$. The flow $\phi^{*}$ on $G / \Gamma$ is ergodic.

I would like to thank the referee for strengthening Theorem A. Specifically, the referee suggested condition $\mathrm{c}(2)$, which is weaker than condition $\mathrm{c}(1)$, and sketched the proof of the sufficiency of condition $c(2)$ (see $\S 8)$. The referee was inspired here by work of Brian Marcus [16].

Theorem A generalizes a classical result in the case where $G$ is $\mathbf{R}^{n}, \Gamma$ is $\mathbf{Z}^{n}$, and $G / \Gamma$ is the $n$-torus (§2). If $G$ and $G^{\prime}$ are nilpotent, and $q: G \rightarrow G /[G, G]$ and $q^{\prime}: G^{\prime} \rightarrow G^{\prime} /\left[G^{\prime}, G^{\prime}\right]$ their abelianization, then the one-parameter subgroups $q \phi$ and $q^{\prime} \phi^{\prime}$ induce toral flows $(q \phi)^{*}$ and $\left(q^{\prime} \phi^{\prime}\right)^{*}$. Theorem A sharpens a result of L. Auslander, F. Hahn, and L. Markus [3, p. 51]. They showed that if $\phi^{*}$ and $\phi^{\prime *}$ are topologically equivalent flows on $G / \Gamma$ and $G^{\prime} / \Gamma^{\prime}$, then the induced toral flows $(q \phi)^{*}$ and $\left(q^{\prime} \phi^{\prime}\right)^{*}$ are affinely equivalent. 
Brian Marcus showed that when $G=G^{\prime}=\operatorname{PSL}(2, \mathbf{R})$ and $\phi$ and $\phi^{\prime}$ are unipotent, then topologically equivalent flows are topologically equivalent by an affine map [16]. Theorem A does not imply Marcus's result, but it does imply a similar result when $G=G^{\prime}=\mathrm{SL}(3, \mathbf{R})(\S 10(\mathrm{~g})$, Example (12)).

Let $G=G^{\prime}=\operatorname{PSL}(2, \mathbf{R})$. Then there exist homogeneous spaces $G / \Gamma$ and $G / \Gamma^{\prime}$ with $G$-induced flows $\phi^{*}$ and $\phi^{\prime *}$, such that $\phi^{*}$ and $\phi^{\prime *}$ are topologically equivalent, but not affinely equivalent, and not topologically conjugate. Furthermore, there is no constant reparameterization of $\phi^{\prime *}$ which would make the flows topologically conjugate (see $\S 10(\mathrm{~h}))$. The limits of Theorem A are also indicated by certain examples where $G$ is solvable ( $(10$, Examples 3, 4, and 5).

The restriction on eigenvalues in Condition (b) insures that isomorphisms of the lattices extend to isomorphisms of the Lie groups, and that $\operatorname{exp:~} L(G) \rightarrow G$ is a diffeomorphism (see $\S 10(\mathrm{f})$ ). The restrictions on $G, G^{\prime}, \Gamma$, and $\Gamma^{\prime}$ in Condition (c) insure that the Mostow rigidity theorem can be applied to show that isomorphisms of the lattices extend to isomorphisms of the Lie groups. For $G$ satisfying Condition (c), the Moore ergodicity theorem [20], completely characterizes those $\phi$ for which $\phi^{*}$ is ergodic. For example, if $G$ is simple and $\phi$ has noncompact closure, then $\phi^{*}$ is ergodic (see $\S 8$ for more detail).

In order to prove Theorem A, in Theorem 1 we abstract and generalize a proof of the toral case. This proof depends on the fact that one-parameter subgroups of $\mathbf{R}^{n}$ diverge from each other. Therefore to apply Theorem 1 , we need Theorem B on the divergence properties of one-parameter subgroups.

$\phi$ is recurrently approached by $\phi^{\prime}$, if there exist sequences

$$
\left\{t_{k} \mid k \in \mathbf{Z}, t_{k} \in \mathbf{R}, \operatorname{sign}(k)=\operatorname{sign}\left(t_{k}\right), t_{k} \rightarrow \pm \infty \text { as } k \rightarrow \pm \infty\right\}
$$

and

$$
\left\{s_{k} \mid k \in \mathbf{Z}, s_{k} \in \mathbf{R}, \operatorname{sign}(k)=\operatorname{sign}\left(s_{k}\right), s_{k} \rightarrow \pm \infty \text { as } k \rightarrow \pm \infty\right\}
$$

such that the set $\left\{\phi\left(t_{k}\right) \phi^{\prime}\left(-s_{k}\right): k \in \mathbf{Z}\right\}$ has compact closure. Equivalently, $\phi$ is recurrently approached by $\phi^{\prime}$, if for positive and negative time, $\phi^{\prime}$ keeps returning to within a bounded distance of $\phi$, where distance is induced from a left or right invariant Riemannian metric. $\phi$ is isolated, if $\phi$ being recurrently approached by $\phi^{\prime}$ implies that $\phi=\phi^{\prime}$ up to sense-preserving reparameterization.

THEOREM B. Let $G$ be a connected Lie group and $\phi$ a one-parameter subgroup.

(a) If $G$ is simply connected and nilpotent, or if $G$ is simply connected and solvable with exp: $L(G) \rightarrow G$ a diffeomorphism, then every $\phi$ of $G$ is isolated.

(b) Let $G$ be semisimple. $\phi(t) \equiv \exp (t X)$ is isolated if $[X, Y]=0$ implies that when $\operatorname{ad}(Y)$ is semisimple then $\operatorname{ad}(Y)$ has some eigenvalue which is not pure imaginary and not 0 . Let the center of $G$ be finite. Then $\phi$ is isolated if there is no compact subgroup in the centralizer of $\phi$.

In $\S 2$, Theorem 1 is proved. Theorem 2 , which demonstrates the isolation of subgroups in the nilpotent and solvable case, is proved in $\S 3$. Theorem 3, which concerns recurrently approaching subgroups of $\mathrm{SL}(n, \mathbf{R})$ and other semisimple groups, is proved in $\S 4$. Theorem 3 requires Proposition 1, which reduces the problem to Proposition 2, which deals with unipotent subgroups. Proposition 1 is in $\S 5$ and Proposition 2 is in $\S 6$. Theorem 4, which demonstrates isolation of subgroups in the semisimple case, is proved in $§ 7$. Theorem 5 , which is Theorem A for the semisimple 
case, is proved in $\S 8 . \S 9$ proves our main results, Theorems A and B. $\S 10$ contains examples, counterexamples, comments, and questions.

This paper is drawn from a doctoral dissertation done at City University of New York. The encouragement and guidance of my advisor Michael Shub were of great help. Much inspiration came from talks with Dennis Sullivan. Martin Moskowitz, John Smillie, and Alphonse Vasquez were generous with their time and expertise. Communications with Jonathan Brezin, Bertram Kostant, G. D. Mostow, and William Parry helped acquaint me with existing work in the field. A doctoral dissertation completion fellowship in 1984-85 from the Sloan Foundation made available the leisure to finish this work. I am grateful for the referee's careful reading of this paper, and for the referee's comments on substance and style.

2. Theorem 1. Theorem 1 generalizes the following proof of the following classical theorem (see $[\mathbf{1 4}$, p. 36] for an alternate proof).

Classical Theorem. For $v$ and $v^{\prime}$ in $\mathbf{R}^{n}$, let $\phi: t \mapsto t v$ and $\phi^{\prime}: t \mapsto t v^{\prime}$ be one-parameter subgroups of $\mathbf{R}^{n}$, and $\phi^{*}:\left(t, x+\mathbf{Z}^{n}\right) \mapsto t v+x+\mathbf{Z}^{n}$ and $\phi^{\prime *}:(t, x+$ $\left.\mathbf{Z}^{n}\right) \mapsto t v^{\prime}+x+\mathbf{Z}^{n}$ the induced flows on the torus $\mathbf{R}^{n} / \mathbf{Z}^{n}$. Then $\phi^{*}$ and $\phi^{\prime *}$ are topologically equivalent if and only if there exists $A$ in $\operatorname{GL}(n, \mathbf{Z})$ and $\alpha>0$ such that $A(v)=\alpha v^{\prime}$.

PROOF. Let $\phi^{*}$ and $\phi^{\prime *}$ be topologically equivalent by a homeomorphism $f$. After translating if necessary, we can assume that $f\left(0+\mathbf{Z}^{n}\right)=\left(0+\mathbf{Z}^{n}\right)$. Let $\tilde{f}$ be the lift of $f$ to $\mathbf{R}^{n}$. Let $A \equiv \tilde{f}_{\mid \mathbf{Z}^{n}} \in \operatorname{GL}(n, \mathbf{Z}) \subset \operatorname{GL}(n, \mathbf{R})$. $\tilde{f}$ takes $\phi$ to $\phi^{\prime}$ bijectively as ordered sets. Hence, $A^{-1} \tilde{f}$ takes $\phi$ to $A^{-1} \phi^{\prime}$ bijectively as ordered sets. $A^{-1} \tilde{f}$ moves points by a bounded amount since $A^{-1} \tilde{f}$ restricted to $\mathbf{Z}^{n}$ is the identity. Since one-parameter subgroups of $\mathbf{R}^{n}$ (i.e. straight lines) diverge, $\phi=A^{-1} \phi^{\prime}$ and $A \phi=\phi^{\prime}$ as ordered sets. That is there exists $\alpha>0$ such that $A(v)=\alpha v^{\prime}$.

The converse implication is clear (see Lemma 1 below). Q.E.D. (Classical Theorem)

The definition of isolation in $\S 1$ and the definitions made below abstract relevant properties from the above proof.

Let $G$ be a connected Lie group and $\Gamma$ a discrete subgroup.

DEFINITION 1. Let $\phi$ be a one-parameter subgroup of $G$. $\phi$ has compact recurrence in $G / \Gamma$ if and only if there is a sequence indexed by the integers

$$
\left\{r_{k} \mid k \in \mathbf{Z}, r_{k} \in \mathbf{R}, \operatorname{sign}\left(r_{k}\right)=\operatorname{sign}(k), r_{k} \rightarrow \pm \infty \text { as } k \rightarrow \pm \infty\right\}
$$

such that there is a compact set in $G / \Gamma$ containing the subset $\left\{\phi\left(r_{k}\right) \Gamma \mid k \in \mathbf{Z}\right\}$ of the trajectory of $e \Gamma$.

EXAMPLES. (a) If $G / \Gamma$ is compact, then every $\phi$ has compact recurrence in $G / \Gamma$.

(b) If $G=\mathrm{SL}(n, \mathbf{R}), \Gamma=\mathrm{SL}(n, \mathbf{Z})$, and $\phi$ is unipotent, then by the Margulis lemma $\phi$ has compact recurrence in $G / \Gamma[8]$.

DEFINITION 2. $(G, \Gamma)$ has the automorphism extension property if and only if every automorphism of $\Gamma$ extends to a Lie group automorphism of $G$.

EXAMPLE. $G=\mathbf{R}^{n}, \Gamma=\mathbf{Z}^{n}$, and $\operatorname{Aut}(\Gamma)=\operatorname{GL}(n, \mathbf{Z}) \subset \mathrm{GL}(n, \mathbf{R})=\operatorname{Aut}(G)$.

DEFINITION 3. Let $e$ be the identity of $G$. Let $p: G \rightarrow G / \Gamma$ be considered as a covering map, where $p$ is the canonical projection. Then $(G, G / \Gamma)$ has the 
homeomorphism lifting property if and only if every homeomorphism from $(G / \Gamma, e \Gamma)$ to $(G / \Gamma, e \Gamma)$ lifts to a homeomorphism from $(G, e)$ to $(G, e)$.

EXAMPLE. If $G$ is simply connected, $(G, G / \Gamma)$ has the homeomorphism lifting property.

THEOREM 1. Let $G$ be a connected Lie group, $\Gamma$ a discrete subgroup, $p: G \rightarrow$ $G / \Gamma$ the canonical projection, $\phi$ and $\phi^{\prime}$ one-parameter subgroups, and $f: G / \Gamma \rightarrow$ $G / \Gamma$ a topological equivalence of the flows $\phi^{*}$ and $\phi^{\prime *}$.

(a) If $\phi$ is isolated, and $\phi$ has compact recurrence in $G / \Gamma$, and $f:(G / \Gamma, e \Gamma) \rightarrow$ $(G / \Gamma, e \Gamma)$ has a lift $\tilde{f}:(G, e) \rightarrow(G, e)$ where $e$ is the identity of $G$, and $\tilde{f}_{\mid \Gamma}$ is the identity on $\Gamma$, then $\phi^{*}$ and $\phi^{\prime *}$ are topologically equivalent by the identity.

(b) In (a) above, if $\tilde{f}_{\mid \Gamma}$ is not assumed to be the identity but $(G, \Gamma)$ has the automorphism extension property, then $\phi^{*}$ and $\phi^{\prime *}$ are topologically equivalent by an affine map preserving $\mathrm{e} \Gamma$.

(c) Let $\Gamma$ be a lattice in $G$, and $(G, G / \Gamma)$ have the homeomorphism lifting property, and $(G, \Gamma)$ have the automorphism extension property. Let $\phi$ be isolated. Then for any other $\phi^{\prime}$ in $G$, the flows $\phi^{*}$ and $\phi^{\prime *}$ are topologically equivalent if and only if they are affinely equivalent.

PROOF. We state without proof the following simple lemma.

LEMMA 1. (a) Let $\bar{a} \bar{A}$ be an affine map from $G / \Gamma$ to $G^{\prime} / \Gamma^{\prime}$. Let $\psi$ be a oneparameter subgroup of $G$. Then $\bar{a} \bar{A}$ is a topological conjugacy and hence a topological equivalence of the flows $\psi^{*}$ and $\left(a(A \psi) a^{-1}\right)^{*}$. (For notation, see below.)

(b) $\psi^{*}$ on $G / \Gamma$ and $\psi^{\prime *}$ on $G^{\prime} / \Gamma^{\prime}$ are topologically equivalent by an affine map if and only if there exist $\alpha>0$ and an affine map $\overline{b B}: G / \Gamma \rightarrow G^{\prime} / \Gamma^{\prime}$ such that for all $t, b(B \psi) b^{-1}(t)=\psi^{\prime}(\alpha t)$.

Notation. For any $\phi$ of $G$ and $a \in G, a \phi a^{-1}(t) \equiv a \phi(t) a^{-1}$.

We return to the proof of Theorem 1. $e$ is the identity.

(a) Since $\phi$ has compact recurrence in $G / \Gamma$, we can pick a sequence indexed by the integers

$$
\left\{t_{k} \mid k \in \mathbf{Z}, t_{k} \in \mathbf{R}, \operatorname{sign}\left(t_{k}\right)=\operatorname{sign}(k), t_{k} \rightarrow \pm \infty \text { as } k \rightarrow \pm \infty\right\}
$$

such that there is a compact set $D$ in $G / \Gamma$ containing $\left\{\phi\left(t^{k}\right) \Gamma \mid k \in \mathbf{Z}\right\}$. Since $\phi$ and $\phi^{\prime}$ are the lifts through $e$ of the trajectories through $e \Gamma$ of the flows $\phi^{*}$ and $\phi^{\prime *}, \tilde{f} \phi=\phi^{\prime}$ as an ordered set. The sequence $\left\{t_{k} \mid k \in \mathbf{Z}\right\}$ determines a sequence indexed by the integers

$$
\left\{s_{k} \mid k \in \mathbf{Z}, s_{k} \in \mathbf{R}, \operatorname{sign}\left(s_{k}\right)=\operatorname{sign}(k), s_{k} \rightarrow \pm \infty \text { as } k \rightarrow \pm \infty\right\}
$$

such that $\tilde{f} \phi\left(t_{k}\right)=\phi^{\prime}\left(s_{k}\right)$.

LEMMA 2. Let $f: G / \Gamma \rightarrow G / \Gamma$ be a homeomorphism with lift $\tilde{f}: G \rightarrow G$ such that $\tilde{f}_{\mid \Gamma}=$ identity on $\Gamma$. Let $D \subset G / \Gamma$ be compact. Then $\left\{x(\tilde{f}(x))^{-1}: x \Gamma \in D\right\}$ is compact.

ProOF OF LEMMA 2. There exists a compact set $\bar{D}, \bar{D} \subset G$, such that if $x \Gamma \in D$, then there exists $\bar{x} \in \bar{D}$ and $\gamma \in \Gamma$ where $\bar{x} \gamma=x . \tilde{f}(\bar{x} \gamma)=\tilde{f}(\bar{x}) \gamma$ since $\tilde{f}_{\mid \Gamma}=$ identity map. If $x \Gamma \in D$, then

$$
x(\tilde{f}(x))^{-1}=\bar{x} \gamma(\tilde{f}(\bar{x} \gamma))^{-1}=\bar{x} \gamma(\tilde{f}(\bar{x}) \gamma)^{-1}=\bar{x}(\tilde{f}(\bar{x}))^{-1} .
$$


Therefore $\left\{x(\tilde{f}(x))^{-1}: x \Gamma \in D\right\} \subset\left\{\bar{x}(\tilde{f}(\bar{x}))^{-1}: \bar{x} \in \bar{D}\right\}$, which is compact. Q.E.D.

$$
\left\{\phi\left(t_{k}\right) \phi^{\prime}\left(-s_{k}\right): k \in \mathbf{Z}\right\}=\left\{\phi\left(t_{k}\right)\left(\tilde{f}\left(\phi\left(t_{k}\right)\right)\right)^{-1}: k \in \mathbf{Z}\right\},
$$

which is contained in $\left\{x(f(x))^{-1}: x \Gamma \in D\right\}$. Therefore, by Lemma $2,\left\{\phi\left(t_{k}\right) \phi^{\prime}\left(-s_{k}\right)\right.$ : $k \in \mathbf{Z}\}$ has compact closure. Thus, $\phi$ is recurrently approached by $\phi^{\prime}$ (see $\S 1$ for definition).

Since $\phi$ is isolated, $\phi=\phi^{\prime}$ up to sense-preserving reparameterization. Therefore, the identity map is a topological equivalence of $\phi^{*}$ and $\phi^{\prime *}$.

(b) $\tilde{f}_{\mid \Gamma}$ is an automorphism of $\Gamma$. By the automorphism extension property of $(G, \Gamma)$, there is an automorphism $A$ of $G$ extending $\left(\tilde{f}_{\mid \Gamma}\right)^{-1}$. By Lemma $1, \phi^{*}$ and $\left(A \phi^{\prime}\right)^{*}$ are topologically equivalent by the map $\bar{A} f:(G / \Gamma, e \Gamma) \rightarrow(G / \Gamma, e \Gamma)$, which has the lift $A \tilde{f}:(G, e) \rightarrow(G, e)$, where $(A \tilde{f})_{\mid \Gamma}$ is the identity on $\Gamma$. Applying Theorem 1(a) shows that $\phi^{*}$ and $\left(A \phi^{\prime}\right)^{*}$ are topologically equivalent by the identity. Therefore, $\phi^{*}$ and $\phi^{\prime *}$ are topologically equivalent by the affine map $\bar{A}^{-1}:(G / \Gamma, e \Gamma) \rightarrow(G / \Gamma, e \Gamma)$.

(c) Let $f: G / \Gamma \rightarrow G / \Gamma$ be a topological equivalence of $\phi^{*}$ and $\phi^{\prime *}$. That $\Gamma$ is a lattice implies that $G / \Gamma$ has finite volume and that $\phi^{*}$ is a measure-preserving flow. Therefore, by the Poincaré recurrence theorem [7, p. 8], we can find a point $a \Gamma$ in some compact set $D$ in $G / \Gamma$, such that the trajectory of $a \Gamma, t \mapsto(\phi(t) a) \Gamma$, keeps returning to $D$ in both positive and negative time.

Let $b \Gamma=f(a \Gamma)$. Then, by Lemma $1, \bar{b}^{-1} f \bar{a}:(G / \Gamma, e \Gamma) \rightarrow(G / \Gamma, e \Gamma)$ is a topological equivalence of $\left(a^{-1} \phi a\right)^{*}$ and $\left(b^{-1} \phi^{\prime} b\right)^{*} . a^{-1} \phi a$ is isolated since $\phi$ is isolated. $a^{-1} \phi a$ has compact recurrence in $G / \Gamma$ since $p\left(a^{-1} \phi a\right)$ is the image under the homeomorphism $\bar{a}^{-1}$ of the recurrent trajectory $t \mapsto(\phi(t) a) \Gamma . \bar{b}^{-1} f \bar{a}$ lifts to a homeomorphism from $(G, e)$ to $(G, e)$ by the homeomorphism lifting property of $(G, G / \Gamma)$. By hypothesis, $(G, \Gamma)$ has the automorphism extension property. Therefore, by applying Theorem $1(\mathrm{~b})$, we obtain that $\left(a^{-1} \phi a\right)^{*}$ and $\left(b^{-1} \phi^{\prime} b\right)^{*}$ are affinely equivalent. Therefore $\phi^{*}$ and $\phi^{\prime *}$ are affinely equivalent. Q.E.D. (Theorem 1)

3. Theorem 2. In the nilpotent case, Theorem 2(a), below, is a corollary of Proposition $2(\S 6)$. However, the proof of Theorem 2 has greater conceptual and geometric clarity.

THEOREM 2. Let $G$ be a connected, simply connected, solvable Lie group, and let the exponential map from the Lie algebra $L(G)$ to $G$ be a diffeomorphism. Let

$$
\left\{t_{k} \mid k \in \mathbf{Z}, t_{k} \in \mathbf{R}, \operatorname{sign}(k)=\operatorname{sign}\left(t_{k}\right), t_{k} \rightarrow \pm \infty \text { as } k \rightarrow \pm \infty\right\}
$$

and

$$
\left\{s_{k} \mid k \in \mathbf{Z}, s_{k} \in \mathbf{R}, \operatorname{sign}(k)=\operatorname{sign}\left(s_{k}\right), s_{k} \rightarrow \pm \infty \text { as } k \rightarrow \pm \infty\right\}
$$

be sequences indexed by the integers. Let $\phi$ and $\phi^{\prime}$ be one-parameter subgroups of $G$.

(a) If $\left\{\phi\left(t_{k}\right) \phi^{\prime}\left(-s_{k}\right): k \in \mathbf{Z}\right\}$ has compact closure, then $\phi=\phi^{\prime}$ up to sensepreserving reparameterization. That is, every one-parameter subgroup of $G$ is isolated (see $§ 1)$.

(b) Let $G$ be nilpotent. If either of the sets

$$
\left\{\phi\left(t_{k}\right) \phi^{\prime}\left(-s_{k}\right): k>0\right\} \quad \text { or } \quad\left\{\phi\left(t_{k}\right) \phi^{\prime}\left(-s_{k}\right): k<0\right\}
$$


has compact closure, then $\phi=\phi^{\prime}$ up to sense-preserving reparameterization. There are counterexamples when $G$ is not nilpotent (see Lemma (b)).

NOTE. Theorem 2(a) applies whenever $G$ is connected, simply connected, and nilpotent, since then the exponential map is a diffeomorphism [12, p. 269]. Theorem 2(b) is a sharper result which applies only to the nilpotent case.

ProOF. Since exp: $L(G) \rightarrow G$ is a diffeomorphism, every connected Lie subgroup is closed.

LEMMA. (a) Theorem 2 holds in case the dimension of $G$ is less than or equal to 3 .

(b) There is a 2-dimensional nonnilpotent counterexample to the conclusion of Theorem 2(b).

We postpone the proof of the lemma to the end of this section.

We proceed by induction on the dimension of $G$.

Assume the result if the dimension of $G$ is $\leq n$, where $n \geq 3$.

Let the dimension of $G$ equal $n+1$. We choose a connected, normal, Lie subgroup $H$ of $G$, having dimension $\geq 1$ and codimension $\geq 2[6$, p. 46]. If $G$ is nilpotent, let $H$ be any one-parameter subgroup contained in the nontrivial center of $G$. Let $p: G \rightarrow H \backslash G$ be the canonical projection.

$H \backslash G$ is a connected, simply connected, solvable Lie group, with exp: $L(H \backslash G) \rightarrow$ $H \backslash G$ a diffeomorphism and with its dimension $\leq n$. If $G$ is nilpotent, then $H \backslash G$ is nilpotent [30, p. $238, \mathbf{6}$, p. 400 , and $\mathbf{2 9}$, p. 7$]$.

Let $X$ and $Y$ be in $L(G)$, and $\phi(t) \equiv \exp (t X)$ and $\phi^{\prime}(t) \equiv \exp (t Y)$, for all $t$ in R. Since $\left\{\phi\left(t_{k}\right) \phi^{\prime}\left(-s_{k}\right): k \in \mathbf{Z}\right\}$ has compact closure, $\left\{\left(p \phi\left(t_{k}\right)\right)\left(p \phi^{\prime}\left(-s_{k}\right)\right): k \in \mathbf{Z}\right\}$ has compact closure. Since by the inductive hypothesis $p \phi$ is isolated, it follows that $p \phi=p \phi^{\prime}$ up to sense-preserving reparameterization. Therefore, there exists $\alpha \in L(H)$ such that $\alpha+X=r Y$ for some $r>0$. Since $L(H)$ is an ideal, $L(H)$ and $X$ generate a subalgebra $L(K)$ which has a dimension one greater than the dimension of $L(H) . L(K)$ is the Lie algebra of $K$, a connected, simply connected, solvable Lie group of dimension $\leq n$, with exp: $L(K) \rightarrow K$ a diffeomorphism. If $G$ is nilpotent, $K$ is nilpotent and isomorphic to $\mathbf{R}^{2}$. Since $X$ and $Y$ are in $L(K)$, $\phi$ and $\phi^{\prime}$ are in $K$. Since $K$ is closed in $G,\left\{\phi\left(t_{k}\right) \phi^{\prime}\left(-s_{k}\right): k \in \mathbf{Z}\right\}$ has compact closure as a subset of $K$. By the inductive hypothesis, $\phi$ is isolated as a subgroup of $K$. Therefore $\phi=\phi^{\prime}$ up to sense-preserving reparameterization.

The same steps prove Theorem 2(b), since when $G$ is nilpotent all the groups involved are nilpotent.

It only remains to give the proof of the lemma.

PROOF OF THE LEMMA. In making computations the following geometric and algebraic consequences of $\phi$ being recurrently approached by $\phi^{\prime}$ are useful. Let $d$ be any metric on $G$ induced from a right or left invariant Riemannian metric. Then $\left\{\phi\left(t_{k}\right) \phi^{\prime}\left(-s_{k}\right): k \in \mathbf{Z}\right\}$ having compact closure is equivalent to $\left\{d\left(\phi\left(t_{k}\right), \phi^{\prime}\left(s_{k}\right)\right): k \in \mathbf{Z}\right\}$ being bounded. Let $G$ be considered as a subgroup of $\mathrm{GL}(n, \mathbf{R})$ in some fixed representation. Let \|\| be any norm on the vector space of $n \times n$ matrices. Then $\left\{\phi\left(t_{k}\right) \phi^{\prime}\left(-s_{k}\right): k \in \mathbf{Z}\right\}$ having compact closure implies that $\left\{\left\|\phi\left(t_{k}\right) \phi^{\prime}\left(-s_{k}\right)\right\|: k \in \mathbf{Z}\right\}$ is bounded.

Let $n$ be the dimension of $G$.

If $n=1$, the result is trivial. 
We examine 3 cases: (i) $n=2$, (ii) $n=3$ and $G$ contains a normal Lie subgroup of dimension one, (iii) $n=3$ and $G$ does not contain a normal Lie subgroup of dimension one.

Case (i). If $n=2, G$ is isomorphic to either the abelian group $\mathbf{R}^{2}$, or to the nonnilpotent matrix group

$$
\left\{\left(\begin{array}{ll}
a & b \\
0 & 1
\end{array}\right) \mid a>0, b \in \mathbf{R}\right\}
$$

which we denote by $S_{0} . L\left(S_{0}\right)$, the Lie algebra of $S_{0}$, is the Lie algebra of matrices

$$
\left\{\left(\begin{array}{ll}
c & d \\
0 & 0
\end{array}\right) \mid c \in \mathbf{R}, d \in \mathbf{R}\right\} \quad[6, \text { p. 44] }
$$

an element of which we denote by $[c, d]$.

Theorem 2 is trivial for $\mathbf{R}^{2}$, where we use the Euclidean metric.

Let us write the elements of $S_{0}$ as ordered pairs $(a, b) \in \mathbf{R}^{+} \times \mathbf{R}$, where $(a, b)\left(a^{\prime}, b^{\prime}\right)=\left(a a^{\prime}, a b^{\prime}+b\right)$.

$$
\exp :[c, d] \mapsto\left(e^{c},(d / c)\left(e^{c}-1\right)\right)
$$

After sense-preserving reparameterization, the three types of one-parameter subgroups of $S_{0}$ are (1) $t \mapsto\left(e^{t}, d\left(e^{t}-1\right)\right),(2) t \mapsto\left(e^{-t}, d\left(e^{-t}-1\right)\right)$, and (3) $t \mapsto(1, t d)$. $d$ is any real number.

Let $\phi$ and $\phi^{\prime}$ be one-parameter subgroups. We show that if $\phi$ is recurrently approached by $\phi^{\prime}$, then $\phi=\phi^{\prime}$ up to sense-preserving reparameterization. The result is clear if $\phi$ is type (1) and $\phi^{\prime}$ is type (2) or type (3), or if $\phi$ is type (3) and $\phi^{\prime}$ is type (1) or type (2). It only remains to check the case when $\phi$ and $\phi^{\prime}$ are type (1).

Let \|\| be a norm on the vector space of $2 \times 2$ matrices.

So, let $\phi(t) \equiv\left(e^{t}, d\left(e^{t}-1\right)\right)$ and $\phi^{\prime}(s) \equiv\left(e^{s}, d^{\prime}\left(e^{s}-1\right)\right)$, for all $t$ and $s$ in $\mathbf{R}$.

$$
\begin{aligned}
\phi(t) \phi^{\prime}(-s) & =\left(e^{t-s}, e^{t} d^{\prime}\left(e^{-s}-1\right)+d\left(e^{t}-1\right)\right) \\
& =\left(e^{t-s}, e^{t}\left(d-d^{\prime}\right)+d^{\prime}\left(e^{t-s}\right)-d\right) .
\end{aligned}
$$

Then $\sup _{k \in \mathbf{Z}}\left\|\phi\left(t_{k}\right) \phi^{\prime}\left(-s_{k}\right)\right\|<\infty$ implies that $e^{\left(t_{k}-s_{k}\right)}$ is bounded. Therefore $d=d^{\prime}$, and thus $\phi=\phi^{\prime}$.

We could also verify that the subgroups are isolated using the metric $d$ induced by the left invariant Riemannian metric $\left(1 / a^{2}\right)(d a \otimes d a+d b \otimes d b)$, which is the Poincaré metric on the upper half-plane. If $(a, b) \in \mathbf{R}^{+} \times \mathbf{R}$ is considered as the complex number $z=b+a i$, it can be computed that

$$
d\left(z_{1}, z_{2}\right)=\log \frac{\left|z_{2}-\bar{z}_{1}\right|+\left|z_{2}-z_{1}\right|}{\left|z_{2}-\bar{z}_{1}\right|-\left|z_{2}-z_{1}\right|} \quad[\mathbf{4}, \text { p. 130]. }
$$

Since the one-parameter subgroups are, after reparameterization, the Euclidean straight lines through $(1,0)$, the result is intuitively clear. See the figure

To get a counterexample to the conclusion of Theorem $2(\mathrm{~b})$ when $G$ is nonnilpotent, consider the subgroups $\phi: t \mapsto\left(e^{t}, e^{t}-1\right)$ and $\phi^{\prime}: s \mapsto\left(e^{s},-e^{s}+1\right)$ of $S_{0}$. (See the diagram.) Reparameterizing the positive directions of the subgroups as 


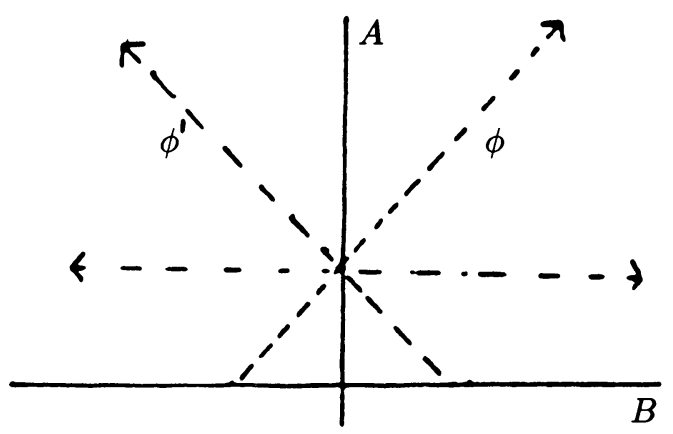

Euclidean half-lines in the upper half-plane, we have for $t>0, \phi: t \mapsto(t+(t+1) i)$ and $\phi^{\prime}: t \mapsto(-t+(t+1) i)$.

$$
\begin{aligned}
\lim _{t \rightarrow \infty} d\left(\phi(t), \phi^{\prime}(t)\right) & =\lim _{t \rightarrow \infty} \log \frac{|2(-t+(t+1) i)|+|-2 t|}{|2(-t+(t+1) i)|-|-2 t|} \\
& =\lim _{t \rightarrow \infty} \log \left(\frac{\sqrt{2 t^{2}+2 t+1}+t}{\sqrt{2 t^{2}+2 t+1}-t}\right)<\infty
\end{aligned}
$$

Therefore taking $t_{k}=s_{k}=k$, we obtain that $\sup _{k>0} d\left(\phi\left(t_{k}\right), \phi^{\prime}\left(s_{k}\right)\right)<\infty$, but $\phi$ is not a sense-preserving reparameterization of $\phi^{\prime}$.

Case (ii). Let $n=3, H$ be a normal Lie subgroup of $G$ of dimension one, and $p: G \rightarrow G / H$. Using the same argument we use in proving the inductive step in Theorem 2, we show that if $\left\{\phi\left(t_{k}\right) \phi^{\prime}\left(-s_{k}\right): k \in \mathbf{Z}\right\}$ has compact closure, then $p \phi=p \phi^{\prime}$ up to sense-preserving reparameterization. This implies that $\phi$ and $\phi^{\prime}$ lie in the same 2-dimensional subgroup of $G$ and thus, by Case (i), that $\phi=\phi^{\prime}$ up to sense-preserving reparameterization. If $G$ is nilpotent, $G / H$ is nilpotent and isomorphic to $\mathbf{R}^{2}$. Therefore, by a similar argument, Theorem 2 (b) follows in this case.

Case (iii). Let $n=3$ and $G$ not have a one-dimensional normal Lie subgroup. Since a nilpotent Lie group has a nontrivial connected center, $G$ is not nilpotent. The commutator subgroup $[G, G]$ must be 2-dimensional, and thus isomorphic to either $\mathbf{R}^{2}$ or $S_{0}$.

We show that $[G, G]$ being isomorphic to $S_{0}$ leads to a contradiction. Let $X$, $Y$, and $Z$ be a basis for $L(G)$, with $X$ and $Y$ in $L([G, G])$. We can assume that $[X, Y]=X[6$, p. 44]. If the range of $\operatorname{ad}(Z)$ on $L([G, G])$ has dimension 0 , then $[G, G]$ would be one-dimensional, which is not so. If the $\operatorname{range}$ of $\operatorname{ad}(Z)$ on $L([G, G])$ has dimension 2 , then $L\left(S_{0}\right)$ has an invertible derivation, which is not so. Therefore let the $\operatorname{range}$ of $\operatorname{ad}(Z)$ on $L([G, G])=\mathbf{R} W$ for some $W \in L([G, G])$, and let $W=\operatorname{ad}(Z)([U, V])$ for some $U$ and $V$ in $L(G)$. $W=\operatorname{ad}(Z)([U, V])=$ $[\operatorname{ad}(z)(U), V]+[U, \operatorname{ad}(Z)(V)]$ which is contained in $\mathbf{R} X$. This implies that $X$ is a one-dimensional ideal of $L(G)$. This is a contradiction, since, by assumption, $G$ has no one-dimensional normal subgroup.

Therefore $[G, G]$ must be isomorphic to $R^{2}$. So $[X, Y]=0$. By a result of Saito, since exp: $L(G) \rightarrow G$ is a diffeomorphism, $\operatorname{ad}(Z)$ has no pure imaginary eigenvalue [29 and 6, p. 400]. It follows that $L(G)$ is isomorphic to the Lie algebra of matrices 
of the form

$$
\left(\begin{array}{cccc}
c & -c \theta & 0 & a \\
c \theta & c & 0 & b \\
0 & 0 & 0 & c \\
0 & 0 & 0 & 0
\end{array}\right), \quad \text { for some fixed } \theta \neq 0
$$

We denote an element of $L(G)$ by $[a, b, c]$. Exponentiating, we see that $G$ is isomorphic to the group of matrices of the form

$$
\left(\begin{array}{cccc}
e^{z} \cos (z \theta) & -e^{z} \sin (z \theta) & 0 & x \\
e^{z} \sin (z \theta) & e^{z} \cos (z \theta) & 0 & y \\
0 & 0 & 0 & z \\
0 & 0 & 0 & 1
\end{array}\right)
$$

That is, $G$ is the semidirect product of $\mathbf{R}^{2}$ by $\mathbf{R}$, where $z \in \mathbf{R}$ acts on $\mathbf{R}^{2}$ by a rotation by $z \theta$ followed by a dilation by $e^{z}$. Writing $G$ as ordered triples, we obtain

$$
(x, y, z)\left(x^{\prime}, y^{\prime}, z^{\prime}\right)=\left((x, y)+e^{z} R_{z \theta}\left(x^{\prime}, y^{\prime}\right), z+z^{\prime}\right)
$$

where $R_{z \theta}$ is counterclockwise rotation of $\mathbf{R}^{2}$ by $z \theta$.

$$
\exp :[a, b, c] \mapsto\left(\left(e^{c} R_{c \theta}-I\right)(u, v), c\right)
$$

where

$$
\left(\begin{array}{l}
u \\
v
\end{array}\right)=\left(\begin{array}{cc}
1 & -\theta \\
\theta & 1
\end{array}\right)^{-1}\left(\begin{array}{l}
a / c \\
b / c
\end{array}\right)
$$

After sense-preserving reparameterization, the three types of one-parameter subgroup of $G$ are

(1) $t \mapsto\left(\left(e^{t} R_{t \theta}-I\right)(u, v), t\right)$,

(2) $t \mapsto\left(\left(e^{-t} R_{-t \theta}-I\right)(u, v),-t\right)$, and

(3) $t \mapsto(t a, t b, 0)$.

$u, v, a$, and $b$ are any real numbers.

We show that if $\phi$ is recurrently approached by $\phi^{\prime}$, then $\phi=\phi^{\prime}$ up to sensepreserving reparameterization. The result is clear if $\phi$ is of type (1) and $\phi^{\prime}$ is of type (2) or type (3), or if $\phi$ is of type (3) and $\phi^{\prime}$ is of type (3) or type (2). It only remains to check the case where $\phi$ and $\phi^{\prime}$ are type (1). \|\| is some norm on the space of $4 \times 4$ matrices.

So let for all $t$ in $\mathbf{R}$,

$$
\begin{aligned}
\phi(t) & \equiv\left(\left(e^{t} R_{t \theta}-I\right)(u, v), t\right), \quad \phi^{\prime}(t) \equiv\left(\left(e^{t} R_{t \theta}-I\right)\left(u^{\prime}, v^{\prime}\right), t\right) . \\
\phi(t) \phi^{\prime}(-s) & =\left(\left(e^{t} R_{t \theta}-I\right)(u, v)+e^{t} R_{t \theta}\left(e^{-s} R_{-s \theta}-I\right)\left(u^{\prime}, v^{\prime}\right), t-s\right) \\
& =\left(e^{t} R_{t \theta}\left(u-u^{\prime}, v-v^{\prime}\right)+e^{t-s} R_{(t-s) \theta}\left(u^{\prime}, v^{\prime}\right)-(u, v), t-s\right) .
\end{aligned}
$$

Since $\sup _{k \in \mathbf{Z}}\left\|\phi\left(t_{k}\right) \phi^{\prime}\left(-s_{k}\right)\right\|<\infty$, it follows that $\sup _{k \in \mathbf{Z}}\left|t_{k}-s_{k}\right|<\infty$. Therefore

$$
\sup _{k \in \mathbf{Z}}\left\|\left(e^{t_{k}}\right) R_{\left(t_{k} \theta\right)}\left(u-u^{\prime}, v-v^{\prime}\right)\right\|<\infty
$$

which implies that $u=u^{\prime}$ and $v=v^{\prime}$. Therefore $\phi=\phi^{\prime}$.

We could also give a geometric argument, using the metric $d$ induced by the left invariant Riemannian metric $e^{-2 z}(d x \otimes d x+d y \otimes d y)+d z \otimes d z$. Q.E.D. (Lemma and Theorem 2) 
4. Theorem 3. In this section we state and prove Theorem 3 , which deals with recurrently approaching one-parameter subgroups of $\mathrm{SL}(n, \mathbf{R})$ and other semisimple Lie groups. Proposition 1 and Proposition 2, on which Theorem 3 depends, are proved in $\S \S 5$ and 6 respectively.

The proof goes roughly as follows. If $\phi$ and $\psi$ are one-parameter subgroups of $\mathrm{SL}(n, \mathbf{R})$, they can be factored as $\phi=E_{1} H_{1} U_{1}$ and $\psi=E_{2} H_{2} U_{2}$ where, for $i=1,2, E_{i}$ has compact closure, $H_{i}$ is positive semisimple and $U_{i}$ is unipotent. In this section we show that if $\phi$ is recurrently approached by $\psi$, then $H_{1} U_{1}$ is recurrently approached by $\mathrm{H}_{2} \mathrm{U}_{2}$. By Proposition $1, \mathrm{H}_{1}=\mathrm{H}_{2}$ up to sense-preserving reparameterization. By Proposition $2, U_{1}=U_{2}$ up to the same reparameterization. This proves Theorem 3.

THEOREM 3. Let $\phi$ and $\psi$ be one-parameter subgroups of $\mathrm{SL}(n, \mathbf{R})$. Suppose that $\phi$ is recurrently approached by $\psi$. Then there is a sense-preserving reparameterization $\psi^{\prime}$ of $\psi$ and a one-parameter subgroup $\chi$ of $\mathrm{SL}(n, \mathbf{R})$ with only positive eigenvalues, such that $\phi(t)=E_{1}(t) \chi(t)$ and $\psi^{\prime}(t)=E_{2}(t) \chi(t)$, where $E_{1}$ and $E_{2}$ are one-parameter subgroups of $\mathrm{SL}(n, \mathbf{R}) . E_{1} \subset \bar{E}_{1} \equiv K_{1}$ and $E_{2} \subset \bar{E}_{2} \equiv K_{2}$, where $K_{1}$ and $K_{2}$ are tori contained in the centralizer of $\chi$. Furthermore, there are one-parameter subgroups $H$ and $U$ of $\mathrm{SL}(n, \mathbf{R})$ such that $\chi(t)=H(t) U(t) . H(t)$ is semisimple with only positive eigenvalues, and $U(t)$ is unipotent. $E_{1}, H$, and $U$ are in each other's centralizer. $E_{2}, H$, and $U$ are in each other's centralizer. Moreover, if $\phi$ and $\psi$ are in $G$, where $G$ is a centerless, semisimple Lie group considered as a subgroup of $\mathrm{SL}(n, \mathbf{R})$ by means of the adjoint representation, then $E_{1}, E_{2}, K_{1}$, $K_{2}, H, U$, and $\chi$ are in $G$.

PROOF.

LEMMA 1. (a) Let \|\| be any norm on $M(n, \mathbf{R})$, the vector space of $n \times n$ matrices. Let $K$ be a compact set in $\mathrm{GL}(n, \mathbf{R})$. Then there exist positive constants $c$ and $C$ such that, for all $g \in M(n, \mathbf{R})$ and $h \in K$,

$$
c\|g\| \leq\|g h\| \leq C\|g\| \quad \text { and } \quad c\|g\| \leq\|h g\| \leq C\|g\| .
$$

(b) Let $\phi, \psi, E_{1}, E_{2}, E_{1} \phi$, and $E_{2} \psi$ be one-parameter subgroups of $\mathrm{GL}(n, \mathbf{R})$, and $K_{1}$ and $K_{2}$ compact sets such that $E_{1} \subset K_{1} \subset \mathrm{GL}(n, \mathbf{R})$ and $E_{2} \subset K_{2} \subset$ $\mathrm{GL}(n, \mathbf{R})$. Then $\phi$ is recurrently approached by $\psi$ if and only if $E_{1} \phi$ is recurrently approached by $E_{2} \psi$.

PROOF. (a) It is enough to prove the result for some particular norm \|\| on $M(n, \mathbf{R})$. So let $\|g\| \equiv \sup _{|v|=1}|g v|$ where || is the Euclidean norm on $\mathbf{R}^{n}$. Then for $g$ and $h$ in $M(n, \mathbf{R}),\|g h\| \leq\|g\|\|h\|$. Let $C \equiv \sup _{h \in K}\|h\|$ and $c \equiv$ $\left(\sup _{h \in K}\left\|h^{-1}\right\|\right)^{-1}$. Then

$$
c\|g\|=c\left\|g h h^{-1}\right\| \leq c\left\|h^{-1}\right\|\|g h\| \leq\|g h\| \leq\|g\|\|h\| \leq C\|g\| .
$$

Also

$$
c\|g\|=c\left\|h^{-1} h g\right\| \leq c\left\|h^{-1}\right\|\|h g\| \leq\|h g\| \leq\|h\|\|g\| \leq C\|g\| .
$$

(b) Lemma 1(b) follows directly from Lemma 1(a) (see $\S 3$, proof of lemma). Q.E.D. (Lemma 1)

We generalize the following decompositions to one-parameter subgroups. 
Complete multiplicative Jordan decomposition. For any element $g \in \mathrm{GL}(n, \mathbf{R})$, there exist unique elements $e, h$, and $u$ in GL $(n, \mathbf{R})$ such that (i) $g=e h u$, (ii) $g, h$, and $u$ commute with each other, and (iii) $u$ is unipotent, $h$ is semisimple with all its eigenvalues positive, and $e$ is semisimple with all its eigenvalues of modulus 1 . It follows that $e$ is contained in a compact connected subgroup. (The letters $e, h$, and $u$ are suggested by the terms elliptic, hyperbolic, and unipotent [12, p. 430].)

Real canonical form. The complete multiplicative Jordan decomposition of an element $g \in \operatorname{GL}(n, \mathbf{R})$ can be read off from the real canonical form of $g$. For an $(n, n)$ real matrix, the real canonical form is the same as Jordan form for the real eigenvalues. For a complex eigenvalue of the form $a+b i$, the blocks consist of $\left(\begin{array}{cc}a & -b \\ b & a\end{array}\right)$ on the diagonal and $\left(\begin{array}{ll}1 & 0 \\ 0 & 1\end{array}\right)$ just below the diagonal $[13$, p. 126].

LEMMA 2. Let $\phi$ be a one-parameter subgroup of $\mathrm{SL}(n, \mathbf{R})$. Then there exist one-parameter subgroups $E, H, U$, and $\chi$ of $\mathrm{SL}(n, \mathbf{R})$, which are in each other's centralizer, and such that $\phi(t)=E(t) H(t) U(t)$ and $\chi(t) \equiv H(t) U(t)$. Furthermore, $E$ is contained in $\bar{E} \equiv K$, a toral subgroup of the centralizer of $\chi, H$ is semisimple and has all its eigenvalues positive, and $U$ is unipotent. $E, H$, and $U$ are the unique subgroups having these properties.

ProOF. Let $\phi(t) \equiv e^{t X}$, for all $t$ in $\mathbf{R}$. Pick a basis for $\mathbf{R}^{n}$ such that $X$ is in real canonical form. Consider the $S+N$ decomposition of $X$. Then $U(t) \equiv e^{t N}$, for all $t$ in $\mathbf{R}$. $E(t) H(t)=e^{t S}$, for all $t$ in $\mathbf{R}$, is the O-P polar decomposition with respect to the basis. Looking at the matrix representation with respect to the chosen basis, we see that the subgroups are in each other's centralizer and have the other required properties. The uniqueness follows from the uniqueness of the complete multiplicative Jordan decomposition for any element in $\operatorname{GL}(n, \mathbf{R})$. Q.E.D. (Lemma 2)

Now let $\phi$ and $\psi$ be the one-parameter subgroups of $\operatorname{SL}(n, \mathbf{R})$ referred to in the statement of Theorem 3. Choosing, as in Lemma $2, E_{1}, \bar{E}_{1} \equiv K_{1}, H_{1}, U_{1}$, $\chi_{1} \equiv H_{1} U_{1}, E_{2}, \bar{E}_{2} \equiv K_{2}, H_{2}, U_{2}$, and $\chi_{2} \equiv H_{2} U_{2}$, we obtain the following:

(i) $E_{1}, H_{1}$, and $U_{1}$ are in each other's centralizer. $E_{2}, H_{2}$, and $U_{2}$ are in each other's centralizer.

(ii) $E_{1} \subset K_{1}$ which is contained in the centralizer of $\chi_{1}$ and $E_{2} \subset K_{2}$ which is contained in the centralizer of $\chi_{2} . K_{1}$ and $K_{2}$ are tori.

(iii) There exist $X_{1}$ and $X_{2}$ such that $\chi_{1}(t)=e^{t X_{1}}$ and $\chi_{2}(t)=e^{t X_{2}}$ where $X_{1}$ and $X_{2}$ have all their eigenvalues real.

(iv) $\phi(t)=E_{1}(t) \chi_{1}(t)$ and $\psi(t)=E_{2}(t) \chi_{2}(t)$.

By using Lemma $1(\mathrm{~b}), \chi_{1}$ is recurrently approached by $\chi_{2}$. Using Proposition $1(\S 5)$ and Proposition $2(\S 6)$, we can show that $\chi_{2}$ is a sense-preserving reparameterization of $\chi_{1}$. Thus after a sense-preserving reparameterization of $\psi$, we can assume that $H_{1}=H_{2} \equiv H, U_{1}=U_{2} \equiv U$, and $\chi_{1}=\chi_{2} \equiv \chi$. This proves the result for $\mathrm{SL}(n, \mathbf{R})$.

Now, let $G$ be a connected, semisimple Lie group considered as a subgroup of $\mathrm{SL}(n, \mathbf{R})$ by means of the adjoint representation. It follows that if, for $g \in G$, $g=e h u$ is the complete multiplicative Jordan decomposition of $g$ as an element in $\mathrm{SL}(n, \mathbf{R})$, then $e, h$, and $u$ are also in $G[\mathbf{1 2}, \mathrm{p} .435]$. Therefore all the subgroups mentioned are in G. Q.E.D. (Theorem 3) 
5. Proposition 1. The following proposition roughly shows that if $\phi$ is recurrently approached by $\psi$ where $\phi$ and $\psi$ have only positive eigenvalues, then up to sense-preserving reparameterization they have the same semisimple parts. This is done by showing that they have the same eigenvalues (Lemma 1) and the same generalized eigenspaces (Lemma 2). Therefore the unipotent part of $\phi$ is approached by the unipotent part of $\psi$. This result is the hypotheses used in Proposition 2 .

Proposition 1. Let $\phi(t) \equiv e^{t X}$ and $\psi(t) \equiv e^{t Y}$, for all $t$ in $\mathbf{R}$, be oneparameter subgroups of $\operatorname{SL}(n, \mathbf{R})$ such that all the eigenvalues of $X$ and $Y$ are real. Let $\phi$ be recurrently approached by $\psi$.

(a) $\phi$ is unipotent if and only if $\psi$ is unipotent.

(b) If $\phi$ and $\psi$ are not unipotent, there is a sense-preserving reparameterization $\psi^{\prime}$ of $\psi$, where for all $t$ in $\mathbf{R} \psi^{\prime}(t) \equiv e^{t Y^{\prime}}$, such that the largest eigenvalue of $X$ and the largest eigenvalue of $Y^{\prime}$ are positive and equal.

(c) Let $\phi$ and $\psi$ be nonunipotent such that $\lambda_{1}=\mu_{1}>0$, where $\lambda_{1}$ is the largest eigenvalue of $X$ and $\mu_{1}$ is the largest eigenvalue of $Y$. Then there are one-parameter subgroups $H, U_{1}$, and $U_{2}$ with the following properties.

(i) $H$ is semisimple with only real eigenvalues. $U_{1}$ and $U_{2}$ are unipotent. For all $t$ and $s$ in $\mathbf{R}, \phi(t)=H(t) U_{1}(t), \psi(t)=H(t) U_{2}(t), H(t) U_{1}(s)=U_{1}(s) H(t)$, and $H(t) U_{2}(s)=U_{2}(s) H(t)$.

(ii) $U_{1}$ is recurrently approached by $U_{2}$.

(iii) $\sup _{k \in \mathbf{Z}}\left|t_{k}-s_{k}\right|<\infty$ where $\left\{t_{k}\right\}$ and $\left\{s_{k}\right\}$ are the sequences for which $\left\{U_{1}\left(t_{k}\right) U_{2}\left(-s_{k}\right): k \in \mathbf{Z}\right\}$ has compact closure (see $\left.\S 1\right)$.

PROOF. We first make some definitions and observations which will also be used in the proof of Proposition 2.

DEFinitions. (1) Let $\operatorname{spec}(X)$ and $\operatorname{spec}(Y)$ be the distinct eigenvalues of $X$ and $Y$ respectively. Let $\mathcal{V}$ and $\mathcal{W}$ be bases of $\mathbf{R}^{n}$ in which $X$ and $Y$ are, respectively, in Jordan form. Let, for all $t$ in $\mathbf{R}, H_{1}(t) \equiv e^{t S_{1}}, U_{1}(t) \equiv e^{t N_{1}}, H_{2}(t) \equiv e^{t S_{2}}$, and $U_{2}(t) \equiv e^{t N_{2}}$ be one-parameter subgroups, where $X=S_{1}+N_{1}$ and $Y=S_{2}+N_{2}$ are the $S+N$ decompositions. It follows that, for all $t$ and $s$ in $\mathbf{R}, H_{1}(t) U_{1}(s)=$ $U_{1}(s) H_{1}(t)$ and $H_{2}(t) U_{2}(s)=U_{2}(s) H_{2}(t)$. For $\lambda \in \operatorname{spec}(X)$ and $\mu \in \operatorname{spec}(Y)$, let

$$
V_{\lambda} \equiv\left\{x \in \mathbf{R}^{n} \mid S_{1} x=\lambda x\right\} \quad \text { and } \quad W_{\mu} \equiv\left\{x \in \mathbf{R}^{n} \mid S_{2} x=\mu x\right\} \text {. }
$$

That is $V_{\lambda}$ and $W_{\mu}$ are generalized eigenspaces of $X$ and $Y$.

$$
\mathbf{R}^{n}=\bigoplus\left\{V_{\lambda} \mid \lambda \in \operatorname{spec}(X)\right\}=\bigoplus\left\{W_{\mu} \mid \mu \in \operatorname{spec}(Y)\right\} .
$$

(2) For $v \in \mathcal{V}$ and $x \in \mathbf{R}^{n}, v^{*} x$ equals the $v$ coordinate of $x$ with respect to the basis $\mathcal{V}$.

(3) Since $\phi$ is recurrently approached by $\psi$, let $\left\{t_{k}\right\}$ and $\left\{s_{k}\right\}$ be the sequences (see $\S 1$ ), such that $\left\{\phi\left(t_{k}\right) \psi\left(-s_{k}\right): k \in \mathbf{Z}\right\}$ has compact closure, and thus $\left\{\left\|\phi\left(t_{k}\right) \psi\left(-s_{k}\right)\right\|: k \in \mathbf{Z}\right\}$ is bounded, where \|\| is any norm on the vector space of $n \times n$ matrices.

(4) Chains in $\mathcal{V}$ and $\mathcal{W}$ are sequences of the form

$$
\left(v, N_{1} v, N_{1}^{2} v, \ldots\right) \text { and }\left(w, N_{2} w, N_{2}^{2} w, \ldots\right)
$$

respectively. $\mathcal{V}$ and $\mathcal{W}$ are partitioned into maximal chains. The first and last elements in a maximal chain are called initial and terminal elements respectively. 
For $v \in \mathcal{V}, \delta(v)$, the degree of $v$, equals $r$ if $N_{1}^{r} v \neq 0$ but $N_{1}^{(r+1)} v=0 . \delta(w)$, for $w \in \mathcal{W}$, is similarly defined. If $v \in \mathcal{V} \cap V_{\lambda}$ and $\delta(v)=r$, then

$$
\phi(t) v=e^{\lambda t}\left(v_{0}+t v_{1}+\cdots+\frac{t^{r}}{r !} v_{r}\right), \quad \text { where } v_{i} \equiv N_{1}^{i} v \text {. }
$$

Similarly, if $w \in \mathcal{W} \cap W_{\mu}$ and $\delta(w)=r$, then

$$
\psi(t) w=e^{\mu t}\left(w_{0}+t w_{1}+\cdots+\frac{t^{r}}{r !} w_{r}\right), \quad \text { where } w_{i} \equiv N_{2}^{i} w
$$

The following observations are used repeatedly.

OBSERVATIONS. (1) Since $\sup _{k \in \mathbf{Z}}\left\|\phi\left(t_{k}\right) \psi\left(-s_{k}\right)\right\|<\infty$, it follows that for $v \in \mathcal{V}$ and $w \in \mathcal{W}, \sup _{k \in \mathbf{Z}}\left|v^{*} \phi\left(t_{k}\right) \psi\left(-s_{k}\right) w\right|<\infty$.

(2) Let $v \in \mathcal{V} \cap V_{\lambda}$ and $w \in \mathcal{W} \cap W_{\mu}$. Then there are chains $\left(v_{0}, \ldots, v_{p}\right)$ and $\left(w_{0}, \ldots, w_{q}\right)$, where $v=v_{p}, w=w_{0}, v_{0}$ is an initial element, and $w_{q}$ is a terminal element.

(3)

$$
\begin{aligned}
v^{*} \psi(t) w & =v^{*} \psi(t) w_{0}=v^{*} e^{\mu t}\left(w_{0}+t w_{1}+\cdots+\frac{t^{q}}{q !} w_{q}\right) \\
& =e^{\mu t}\left(\left(v^{*} w_{0}\right)+t\left(v^{*} w_{1}\right)+\cdots+\frac{t^{q}}{q !}\left(v^{*} w_{q}\right)\right)
\end{aligned}
$$

(4)

$$
\begin{aligned}
v^{*} \phi(t) w & =v_{p}^{*} \phi(t) w=v_{p}^{*} \phi(t)\left(\left(v_{0}^{*} w\right) v_{0}+\left(v_{1}^{*} w\right) v_{1}+\cdots+\left(v_{p}^{*} w\right) v_{p}\right) \\
& =e^{\lambda t}\left(\frac{t^{p}}{p !}\left(v_{0}^{*} w\right)+\frac{t^{p-1}}{(p-1) !}\left(v_{1}^{*} w\right)+\cdots+\left(v_{p}^{*} w\right)\right) .
\end{aligned}
$$

(5) In particular, if $v_{i}^{*} w=0$ for $i<p$ (equivalently, if $v$ is of maximal degree in the set $\left.\left\{v \in V_{\lambda} \cap \mathcal{V} \mid v^{*} w \neq 0\right\}\right)$, then $v^{*} \phi(t) w=e^{\lambda t}\left(v^{*} w\right)$.

(6) Symmetrically, it is also the case that $\psi$ is recurrently approached by $\phi$, and $\sup _{k \in \mathbf{Z}}\left\|\psi\left(s_{k}\right) \phi\left(-t_{k}\right)\right\|<\infty$. (The reason is that the closure of $\left\{\psi\left(s_{k}\right) \phi\left(-t_{k}\right) \mid k \in\right.$ $\mathbf{Z}\}$ is compact because the closure of $\left\{\phi\left(t_{k}\right) \psi\left(-s_{k}\right) \mid k \in \mathbf{Z}\right\}$ is compact.)

PROOF OF PROPOSITION 1(a). We show first that if $\phi$ is unipotent, then $\psi$ is unipotent.

Assume that $\phi$ is unipotent and $\psi$ is not unipotent. Let $\mu$ be a nonzero eigenvalue of $Y$, with eigenvector $w \in \mathcal{W}$. Let $v$ be of maximal degree in the set $\left\{v \in V_{0} \cap \mathcal{V}=\right.$ $\left.\mathcal{V} \mid v^{*} w \neq 0\right\}$. Then

$$
v^{*} \phi(t) \psi(-s) w=v^{*} \phi(t) e^{-\mu s} w=e^{-\mu s} v^{*} \phi(t) w=e^{-\mu s}\left(v^{*} w\right)
$$

(see Observation 5). Since $\sup _{k \in \mathbf{Z}}\left|v^{*} \phi\left(t_{k}\right) \psi\left(-s_{k}\right) w\right|<\infty, v^{*} w=0$, which is a contradiction.

By symmetry (Observation 6), if $\psi$ is unipotent then $\phi$ is unipotent.

PROOF OF PROPOSITION 1(b). Since $X$ and $Y$ are in the Lie algebra of $\operatorname{SL}(n, \mathbf{R})$, it follows that $\operatorname{trace}(X)=\operatorname{trace}(Y)=0$. Since $\operatorname{spec}(X) \neq\{0\}$ and $\operatorname{spec}(Y) \neq\{0\}$, it follows that $\lambda_{1}$, the largest eigenvalue of $X$, and $\mu_{1}$, the largest eigenvalue of $Y$, are positive. Let $Y^{\prime} \equiv\left(\lambda_{1} / \mu_{1}\right) Y$.

PROOF OF PROPOSITION 1(c). We need the following two lemmas. 
LEMMA 1. If $\phi$ and $\psi$ are nonunipotent and $\lambda_{1}=\mu_{1}>0$, then $\operatorname{spec}(X)=$ $\operatorname{spec}(Y)$ and $\sup _{k \in \mathbf{Z}}\left|t_{k}-s_{k}\right|<\infty$.

PROOF. The proof is postponed.

LEMMA 2. If $\phi$ and $\psi$ are nonunipotent, then, for all $\lambda \in \operatorname{spec}(X)=\operatorname{spec}(Y)$, $V_{\lambda} \supset W_{\lambda}$.

PROOF. The proof is postponed (see end of $\S 5$ ).

Lemma 2 and the fact that $\mathbf{R}^{n}=\bigoplus V_{\lambda}=\bigoplus W_{\lambda}$ imply, by dimensional considerations, that for all $\lambda, V_{\lambda}=W_{\lambda}$. Therefore, for all $\lambda, S_{1}$ and $S_{2}$ agree on $V_{\lambda}$. Therefore $S_{1}=S_{2}$, and hence $H_{1}=H_{2}$. By letting $H \equiv H_{1}=H_{2}$, Proposition $1(\mathrm{c}(\mathrm{i}))$ is proved.

$$
\begin{aligned}
\infty>\sup _{k \in \mathbf{Z}}\left\|\phi\left(t_{k}\right) \psi\left(-s_{k}\right)\right\| & =\sup _{k \in \mathbf{Z}}\left\|H\left(t_{k}\right) U_{1}\left(t_{k}\right) U_{2}\left(-s_{k}\right) H\left(-s_{k}\right)\right\| \\
& =\sup _{k \in \mathbf{Z}}\left\|U_{1}\left(t_{k}\right) U_{2}\left(-s_{k}\right) H\left(t_{k}-s_{k}\right)\right\| .
\end{aligned}
$$

Since, by Lemma $1, \sup _{k \in \mathbf{Z}}\left|t_{k}-s_{k}\right|<\infty$, it follows that $\sup _{k \in \mathbf{Z}}\left\|U_{1}\left(t_{k}\right) U_{2}\left(-s_{k}\right)\right\|<$ $\infty$ (see Theorem 3 , Lemma 1 ). So $U_{1}$ is recurrently approached by $U_{2}$.

To complete the proof of Proposition 1, it only remains to prove Lemma 1 and Lemma 2.

PROOF OF LEMMA 1. Lemma 1 requires the following sublemma.

Sublemma. (a) For all $\mu \in \operatorname{spec}(Y)$ there exists $\lambda \in \operatorname{spec}(X)$, such that $\sup _{k \in \mathbf{Z}}\left(\lambda t_{k}-\mu s_{k}\right)<\infty$.

(b) There is a bijection $\rho$ from $\operatorname{spec}(X)$ to $\operatorname{spec}(Y)$ such that if $\mu=\rho(\lambda)$, then $\sup _{k \in \mathbf{Z}}\left|\lambda t_{k}-\mu s_{k}\right|<\infty$.

Proof of Sublemma. (a) Let $w$ be an eigenvector associated to $\mu$, and pick $v$ of maximal degree from $\left\{v \in \mathcal{V} \mid v^{*} w \neq 0\right\}$. For some $\lambda$, we have $v \in V_{\lambda}$. Then

$$
v^{*} \phi(t) \psi(-s) w=v^{*} \phi(t) e^{-\mu s} w=e^{-\mu s} v^{*} \phi(t) w=e^{-\mu s} e^{\lambda t}\left(v^{*} w\right) .
$$

$\sup _{k \in \mathbf{Z}}\left|v^{*} \phi\left(t_{k}\right) \psi\left(-s_{k}\right) w\right|<\infty$ implies that $\sup _{k \in \mathbf{Z}}\left(\lambda t_{k}-\mu s_{k}\right)<\infty$.

(b) By symmetry (Observation 6), for all $\lambda^{\prime} \in \operatorname{spec}(X)$ there exists $\mu^{\prime} \in \operatorname{spec}(Y)$ such that $\sup _{k \in \mathbf{Z}}\left(\mu^{\prime} s_{k}-\lambda^{\prime} t_{k}\right)<\infty$. Start with $\mu$, pick $\lambda$, set $\lambda^{\prime}=\lambda$, and then pick $\mu^{\prime}$. By addition, $\sup _{k \in \mathbf{Z}}\left(\lambda t_{k}-\mu s_{k}\right)<\infty$ and $\sup _{k \in \mathbf{Z}}\left(\mu^{\prime} s_{k}-\lambda^{\prime} t_{k}\right)<\infty$ imply that $\mu^{\prime}=\mu$. Start with $\lambda^{\prime}$, pick $\mu^{\prime}$, set $\mu=\mu^{\prime}$, and then pick $\lambda$. By adding the same formulas, we see that $\lambda=\lambda^{\prime}$. This establishes the existence of a bijection $\rho$. If $\mu=\rho(\lambda), \sup _{k \in \mathbf{Z}}\left(\lambda t_{k}-\mu s_{k}\right)<\infty$ and $\sup _{k \in \mathbf{Z}}\left(\mu s_{k}-\lambda t_{k}\right)<\infty$ together imply that $\sup _{k \in \mathbf{Z}}\left|\lambda t_{k}-\mu s_{k}\right|<\infty$. Q.E.D. (Sublemma)

We return to the proof of Lemma 1 .

Let $\mu=\rho(\lambda) \sup _{k \in \mathbf{Z}}\left|\lambda t_{k}-\mu s_{k}\right|<\infty$ implies that $\rho(\lambda)=0$ if and only if $\lambda=0$. While if $\lambda \neq 0,(\lambda / \mu)=\lim _{k \rightarrow \infty}\left(s_{k} / t_{k}\right)=\lim _{k \rightarrow-\infty}\left(s_{k} / t_{k}\right)$, where the limits, in fact, exist. By the conditions on $\left\{t_{k}\right\}$ and $\left\{s_{k}\right\}$ (see $\left.\S 1\right), \lim _{k \rightarrow \infty}\left(s_{k} / t_{k}\right) \geq 0$, and so $\rho$ must be an order preserving bijection. So $\rho\left(\lambda_{1}\right)$ equals $\mu_{1}$, which by assumption equals $\lambda_{1}$. So, for all $\mu$ such that $\mu=\rho(\lambda)$, since $\lambda / \mu=\lim _{k \rightarrow \infty}\left(s_{k} / t_{k}\right)=\lambda_{1} / \mu_{1}=$ 1 , it follows that $\lambda=\mu$. Therefore $\rho$ is the identity and thus $\operatorname{spec}(X)=\operatorname{spec}(Y)$. Picking $\lambda=\lambda_{1}$ and $\mu=\rho\left(\lambda_{1}\right)=\mu_{1}, \sup _{k \in \mathbf{Z}}\left|\lambda t_{k}-\mu s_{k}\right|<\infty$ implies that $\sup _{k \in \mathbf{Z}}\left|t_{k}-s_{k}\right|<\infty$. Q.E.D. (Lemma 1)

PROOF OF LEMMA 2. The proof is by contradiction. 
Suppose there exists $\lambda$ such that it is not the case that $V_{\lambda} \supset W_{\lambda}$.

(i) Pick $w$ of minimal degree in the set $\left\{w \in W_{\lambda} \mid w \notin V_{\lambda}\right\}$. There is a maximal chain $\left(w=w_{0}, w_{1}, \ldots, w_{q}\right)$ in $W$.

(ii) Pick $v$ of maximal degree in the set $\left\{v \in\left(\mathcal{V}-V_{\lambda}\right) \mid v^{*} w \neq 0\right\} . v \in V_{\lambda^{\prime}}$ for some $\lambda^{\prime} \neq \lambda$.

$$
v^{*} \phi(t) \psi(-s) w=v^{*} \phi(t) e^{-\lambda s}\left(w-s w_{1}+\frac{s^{2}}{2 !} w_{2} \cdots\right)=e^{-\lambda s} v^{*} \phi(t) w,
$$

by our choice of $w \cdot e^{-\lambda s} v^{*} \phi(t) w=e^{-\lambda s} e^{\lambda^{\prime} t}\left(v^{*} w\right)$, by our choice of $v$ (Observation 5). Since $\sup _{k \in \mathbf{Z}}\left|v^{*} \phi\left(t_{k}\right) \psi\left(-s_{k}\right) w\right|<\infty$, it follows that $\sup _{k \in \mathbf{Z}}\left(\lambda^{\prime} t_{k}-\lambda s_{k}\right)<\infty$.

If $\lambda=0$, then $\lambda^{\prime}=0$, which is a contradiction since $\lambda^{\prime} \neq \lambda$.

If $\lambda>0$,

$$
\lim _{k \rightarrow-\infty}\left(s_{k} / t_{k}\right) \leq \lambda^{\prime} / \lambda \leq \lim _{k \rightarrow \infty}\left(s_{k} / t_{k}\right) .
$$

Since, by Lemma 1 , both limits are equal to 1 , it follows that $\lambda^{\prime}=\lambda$, which is a contradiction.

Similarly, if $\lambda<0$, we get the contradiction $\lambda^{\prime}=\lambda$.

Therefore, $V_{\lambda} \supset W_{\lambda}$. Q.E.D. (Lemma 2 and Proposition 1)

\section{Proposition 2.}

PROPOSITION 2. Let $\phi$ and $\psi$ be unipotent one-parameter subgroups of $\mathrm{SL}(n, \mathbf{R})$. Let $\phi$ be recurrently approached by $\psi$, where $\left\{t_{k} \mid k \in \mathbf{Z}\right\}$ and $\left\{s_{k} \mid k \in \mathbf{Z}\right\}$ are the sequences chosen such that $\left\{\phi\left(t_{k}\right) \psi\left(-s_{k}\right): k \in \mathbf{Z}\right\}$ has compact closure. Then $\psi$ is a sense-preserving reparameterization of $\phi$. Furthermore, if

$$
\sup _{k \in \mathbf{Z}}\left|t_{k}-s_{k}\right|<\infty
$$

then $\psi=\phi$.

Proof. For all $t$ in $\mathbf{R}$, let $\phi(t) \equiv e^{t X}$ and $\psi(t) \equiv e^{t Y}$, where $X$ and $Y$ are nilpotent. We use again the definitions and observations made in proving Proposition $1(\S 5)$, with the difference that here $X$ and $Y$ have no semisimple part and $\operatorname{spec}(X)=\operatorname{spec}(Y)=\{0\}$. For convenience, we restate Observations 1-5 in this context.

OBservations. ( $\left.1^{\prime}\right)$ For all $v \in \mathcal{V}$ and $w \in \mathcal{W}, \sup _{k \in \mathbf{Z}}\left|v^{*} \phi\left(t_{k}\right) \psi\left(-s_{k}\right) w\right|<\infty$.

$\left(2^{\prime}\right)$ Let $v \in \mathcal{V}$ and $w \in \mathcal{W}$. There exist chains $\left(v_{0}, \ldots, v_{p}\right)$ and $\left(w_{0}, \ldots, w_{q}\right)$ where $v=v_{p}, w=w_{0}, v_{0}$ is an initial element, and $w_{q}$ is a terminal element.

$\left(3^{\prime}\right)$

$$
v^{*} \psi(t) w=v_{p}^{*} \psi(t) w_{0}=\left(v_{p}^{*} w_{0}\right)+t\left(v_{p}^{*} w_{1}\right)+\cdots+\frac{t^{q}}{q !}\left(v_{p}^{*} w_{q}\right)
$$

$$
v^{*} \phi(t) w=v_{p}^{*} \phi(t) w_{0}=\frac{t^{p}}{p !}\left(v_{0}^{*} w_{0}\right)+\frac{t^{p-1}}{(p-1) !}\left(v_{1}^{*} w_{0}\right)+\cdots+t\left(v_{p-1}^{*} w_{0}\right)+\left(v_{p}^{*} w_{0}\right) \text {. }
$$

$\left(5^{\prime}\right)$ In particular, $v^{*} \phi(t) w=v_{p}^{*} w_{0}$ if $v_{i}^{*} w_{0}=0$ for $i<p$, and $v^{*} \phi(t) w=$ $\left(v_{p}^{*} w_{0}\right)+t\left(v_{p-1}^{*} w_{0}\right)$ if $v_{i}^{*} w_{0}=0$ for $i<p-1$.

Clearly $\phi$ is the identity if and only if $\psi$ is the identity, and in that case Proposition 2 trivially follows. So assume $\phi$ is not the identity and $\psi$ is not the identity. The following two lemmas directly prove Proposition 2. 
LEMMA 1. (a) There is a real number $\alpha>0$ such that $\sup _{k \in \mathbf{Z}}\left|\alpha t_{k}-s_{k}\right|<\infty$.

(b) Let $\psi^{\prime}(t) \equiv \psi(\alpha t)$, for all $t$ in $\mathbf{R}$. Then $\phi$ is recurrently approached by $\psi^{\prime}$. The sequences $\left\{t_{k}^{\prime}\right\}$ and $\left\{s_{k}^{\prime}\right\}$, chosen so that $\left\{\phi\left(t_{k}^{\prime}\right) \psi^{\prime}\left(-s_{k}^{\prime}\right): k \in \mathbf{Z}\right\}$ has compact closure, have the property that $\sup _{k \in \mathbf{Z}}\left|t_{k}^{\prime}-s_{k}^{\prime}\right|<\infty$.

LEMMA 2. If $\sup _{k \in \mathbf{Z}}\left|t_{k}-s_{k}\right|<\infty$, then $\phi=\psi$.

It remains to prove Lemma 1 and Lemma 2 .

PROOF OF LEMMA 1 . We need the following sublemma.

Sublemma. (a) Let $v \in \mathcal{V}$ and $w \in \mathcal{W}$, such that $\delta(w)=0$. If $\delta(v)>\delta(w)$, then $v^{*} w=0$.

(b) Let $v \in \mathcal{V}$ and $w \in \mathcal{W}$ such that $\delta(w)=1$. If $\delta(v)>\delta(w)$, then $v^{*} w=0$.

(c) There are chains $\left(v_{0}, v_{1}\right)$ in $\mathcal{V}$ and $\left(w_{0}, w_{1}\right)$ in $\mathcal{W}$ where $v_{1}$ and $w_{1}$ are eigenvectors (i.e. $\delta(v)=\delta(w)=0$ ), such that $v_{1}$ is of maximal degree in the set $\left\{v \in \mathcal{V} \mid v^{*} w_{1} \neq 0\right\}$ and either $v_{0}^{*} w_{0}=0$ or $v_{0}$ is of maximal degree in the set $\left\{v \in \mathcal{V} \mid v^{*} w_{0} \neq 0\right\}$.

ProOF OF SUblemma. (a) Let $\delta\left(w_{0}\right)=0$. Let $v_{0}$ be of maximal degree in $\left\{v \in \mathcal{V} \mid v^{*} w_{0} \neq 0\right\}$. If $\delta\left(v_{0}\right) \neq 0$, then there is a chain $\left(v_{0}, v_{1}\right)$ in $\mathcal{V}$.

$$
v_{1}^{*} \phi(t) \psi(-s) w_{0}=v_{1}^{*} \phi(t) w_{0}=\left(v_{1}^{*} w_{0}\right)+t\left(v_{0}^{*} w_{0}\right)
$$

(Observation $\left.5^{\prime}\right)$. Since $\sup _{k \in \mathbf{Z}}\left|v_{1}^{*} \phi\left(t_{k}\right) \psi\left(-s_{k}\right) w_{0}\right|<\infty$, it follows that $v_{0}^{*} w_{0}=0$, which is a contradiction. So $\delta\left(v_{0}\right)=0$.

(b) Let $\delta\left(w_{0}\right)=1$. Then there is a chain $\left(w_{0}, w_{1}\right)$ in $\mathcal{W}$. Let $v_{0}$ be of maximal degree in the set $\left\{v \in \mathcal{V} \mid v^{*} w_{0} \neq 0\right\}$. If $\delta\left(v_{0}\right)>1$, there is a chain $\left(v_{0}, v_{1}\right)$ in $\mathcal{V}$.

$$
v_{1}^{*} \phi(t) \psi(-s) w_{0}=v_{1}^{*} \phi(t)\left(w_{0}-s w_{1}\right)=v_{1}^{*} \phi(t) w_{0}-s v_{1}^{*} \phi(t) w_{1}=v_{1}^{*} \phi(t) w_{0},
$$

since $\delta\left(w_{1}\right)=0, \delta\left(v_{1}\right)>0$, and thus (a) applies (Observation $\left.5^{\prime}\right)$.

$$
v_{1}^{*} \phi(t) w_{0}=v_{1}^{*} w_{0}+t\left(v_{0}^{*} w_{0}\right) \quad\left(\text { Observation } 5^{\prime}\right) .
$$

Since $\sup _{k \in \mathbf{Z}}\left|v_{1}^{*} \phi\left(t_{k}\right) \psi\left(-s_{k}\right) w_{0}\right|<\infty, v_{0}^{*} w_{0}=0$, which is a contradiction. Therefore, $\delta\left(v_{0}\right) \leq 1$. (We note in passing, that continuing inductively we could show that for all $v \in \mathcal{V}$ and $w \in \mathcal{W}$, if $\delta(v)>\delta(w)$, then $v^{*} w=0$.)

(c) Since we are assuming $\psi$ is not the identity, there is a chain $\left(w_{0}, w_{1}\right)$ in $\mathcal{W}$ where $w_{1}$ is an eigenvector (i.e. $\delta\left(w_{1}\right)=0$ ). Pick $v_{1} \in \mathcal{V}$ such that $v_{1}^{*} w_{1} \neq 0$. By part (a) above, $\delta\left(v_{1}\right)=0$ and $v_{1}$ is of maximal degree in the set $\left\{v \in \mathcal{V} \mid v^{*} w_{1} \neq 0\right\}$.

If there is no chain of the form $\left(v_{0}, v_{1}\right)$, then

$$
v_{1}^{*} \phi(t) \psi(-s) w_{0}=v_{1}^{*} \phi(t)\left(w_{0}-s w_{1}\right)=v_{1}^{*} w_{0}-s\left(v_{1}^{*} w_{1}\right)
$$

(see Observation $5^{\prime}$ ). Since $\sup _{k \in \mathbf{Z}}\left|v_{1}^{*} \phi\left(t_{k}\right) \psi\left(-s_{k}\right) w_{0}\right|<\infty, v_{1}^{*} w_{1}=0$, which is a contradiction.

So let $\left(v_{0}, v_{1}\right)$ be a chain in $\mathcal{V}$. By part (b) above, either $v_{0}^{*} w_{0}=0$ or $v_{0}$ is of maximal degree in the set $\left\{v \in \mathcal{V} \mid v^{*} w_{0} \neq 0\right\}$. Q.E.D. (Sublemma)

We return to the proof of Lemma 1.

(a) Let $\left(v_{0}, v_{1}\right)$ and $\left(w_{0}, w_{1}\right)$ be the chains referred to in Sublemma (c).

$$
\begin{aligned}
v_{1}^{*} \phi(t) \psi(-s) w_{0} & =v_{1}^{*} \phi(t)\left(w_{0}-s w_{1}\right) \\
& =v_{1}^{*} \phi(t) w_{0}-s v_{1}^{*} \phi(t) w_{1} \\
& =\left(v_{1}^{*} w_{0}+t\left(v_{0}^{*} w_{0}\right)\right)-s\left(v_{1}^{*} w_{1}\right)
\end{aligned}
$$


(by the choice of $v_{0}$ and $v_{1}$, and by Observation $\left.5^{\prime}\right)$. Let $\alpha \equiv\left(v_{0}^{*} w_{0}\right) /\left(v_{1}^{*} w_{1}\right)$. Since $\sup _{k \in \mathbf{Z}}\left|v_{1}^{*} \phi\left(t_{k}\right) \psi\left(-s_{k}\right) w_{0}\right|<\infty$, it follows that $\sup _{k \in \mathbf{Z}}\left|\alpha t_{k}-s_{k}\right|<\infty$ and $\alpha>0$.

(b) Let $\psi^{\prime}(t) \equiv \psi(\alpha t)$ for all $t$ in $\mathbf{R}$, and let $t_{k}^{\prime} \equiv t_{k}$, and $s_{k}^{\prime} \equiv s_{k} / \alpha$.

$$
\left\{\phi\left(t_{k}^{\prime}\right) \psi^{\prime}\left(-s_{k}^{\prime}\right): k \in \mathbf{Z}\right\}=\left\{\phi\left(t_{k}\right) \psi\left(-s_{k}\right): k \in \mathbf{Z}\right\},
$$

which has compact closure. So $\phi$ is recurrently approached by $\psi^{\prime}$, with the sequences being $\left\{t_{k}^{\prime}\right\}$ and $\left\{s_{k}^{\prime}\right\}$. Furthermore

$$
\sup _{k \in \mathbf{Z}}\left|t_{k}^{\prime}-s_{k}^{\prime}\right|=\sup _{k \in \mathbf{Z}}\left|t_{k}-s_{k} / \alpha\right|<\infty .
$$

\section{Q.E.D. (Lemma 1)}

ProOf OF Lemma 2. By assumption, $\left\{\phi\left(t_{k}\right) \psi\left(-s_{k}\right): k \in \mathbf{Z}\right\}$ has compact closure. Since, by Lemma $1,\left\{t_{k}-s_{k}: k \in \mathbf{Z}\right\}$ is bounded, $\left\{\psi\left(s_{k}-t_{k}\right): k \in \mathbf{Z}\right\}$ has compact closure. Since

$$
\phi\left(t_{k}\right) \psi\left(-t_{k}\right)=\phi\left(t_{k}\right) \psi\left(-s_{k}\right) \psi\left(s_{k}-t_{k}\right),
$$

$\left\{\phi\left(t_{k}\right) \psi\left(-t_{k}\right): k \in \mathbf{Z}\right\}$ has compact closure and is bounded in any norm on the vector space of $n \times n$ matrices. But every component of the matrix valued function $\phi(t) \psi(-t)$ is a polynomial in $t$ and therefore either diverges to infinity or is constant. Therefore each component of the matrix valued function $\phi(t) \psi(-t)$ is constant. Since $\phi(0) \psi(0)$ is the identity, it follows that, for all $t, \phi(t) \psi(-t)$ is the identity, and that, for all $t, \phi(t)=\psi(t)$. Q.E.D. (Lemma 2 and Proposition 2)

7. Theorem 4. Theorem 4 is a consequence of Theorem 3.

THEOREM 4. Let $\phi(t)=\exp (t X)$, for all $t$ in $\mathbf{R}$, be a one-parameter subgroup of a connected, semisimple Lie group G. If Condition (i) or Condition (ii) holds, then $\phi$ is isolated. If $G$ has finite center, Condition (i) and Condition (ii) are equivalent.

CONDITION (i). $G$ has finite center, and there is no compact connected Lie subgroup in the centralizer of $\phi$.

CONDITION (ii). For each $Y$ in the centralizer of $X$ (i.e. $[X, Y]=0$ ), when $\operatorname{ad}(Y)$ is semisimple then $\operatorname{ad}(Y)$ has some eigenvalue which is not pure imaginary and not 0 .

ProOF. Let $Z$ be the center of $G$, and let $p: G \rightarrow G / Z$ be the canonical projection.

(a) $Z$ is trivial-Condition (i) implies $\phi$ is isolated. Since $G$ is centerless, the adjoint representation of $G$ is faithful $[\mathbf{1 2}$, p. 129]. Therefore we can consider $G$ to be a Lie subgroup of $\operatorname{GL}(n, \mathbf{R})$, where $n$ is the dimension of $G$. Since $G$ is semisimple, the commutator subgroup $[G, G]$ equals $G$, which implies that $G$ is a Lie subgroup of $\operatorname{SL}(n, \mathbf{R})[\mathbf{3 0}$, p. 243]. Also, since $G$ is semisimple, $G$ is a closed Lie subgroup of $\mathrm{SL}(n, \mathbf{R})[\mathbf{1 2}$, p. 152].

Let $\phi$ be recurrently approached by $\psi$, a one-parameter subgroup of $G$. Since $G$ is closed in $\operatorname{SL}(n, \mathbf{R}), \phi$ is recurrently approached by $\psi$, where $\phi$ and $\psi$ are considered as subgroups of $\mathrm{SL}(n, \mathbf{R})$. Applying Theorem 3 , we see that there exists a sensepreserving reparameterization $\psi^{\prime}$ of $\psi$ such that, for all $t$ in $\mathbf{R}, \phi(t)=E_{1}(t) H(t) U(t)$ and $\psi^{\prime}(t)=E_{2}(t) H(t) U(t)$, where $E_{1}, E_{2}, H$, and $U$ are subgroups of $G$ with the properties indicated in Theorem 3. 
If Condition (i) holds, since there is no compact, connected subgroup in the centralizer of $\phi$, it follows that $E_{1}$ and thus also $E_{2}$ are trivial. Therefore $\phi=\psi^{\prime}$. This shows that $\phi$ is isolated.

(b) $Z$ is nontrivial Condition (i) implies $\phi$ is isolated. Let $\phi$ be recurrently approached by $\psi$. Then $p \phi$ is recurrently approached by $p \psi$. Since $Z$ is finite, Condition (i) holding for $\phi$ implies Condition (i) holding for $p \phi$. Therefore, by (a), $p \phi$ is isolated and thus $p \phi=p \psi$. Since $Z$ is discrete, $p$ is a covering map and thus one-parameter subgroups of $G / Z$ iift uniquely to one-parameter subgroups of $G$. Therefore $\phi=\psi$. Thus, $\phi$ is isolated.

(c) Condition (ii) implies $\phi$ is isolated. For $W$ in the Lie algebra of $\operatorname{SL}(n, \mathbf{R})$, the one-parameter subgroup $t \mapsto e^{t W}$ has compact closure if and only if $W$ is semisimple and every eigenvalue of $W$ is pure imaginary or 0 . Therefore Condition (ii) is equivalent to there being no compact connected subgroup in the centralizer in $\operatorname{Ad}(G / Z)$ of the one-parameter subgroup $t \mapsto \exp (t(\operatorname{ad}(X)))$. Since Ad: $G / Z \rightarrow \operatorname{SL}(n, \mathbf{R})$ is faithful and $\operatorname{Ad}(G / Z)$ is closed in $\operatorname{SL}(n, \mathbf{R})$, this is equivalent to Condition (i) holding for $p \phi: t \mapsto \exp _{(G / Z)} t X$. Since $G / Z$ is centerless, $p \phi$ is isolated ((a) above). Since one-parameter subgroups of $G / Z$ lift uniquely to one-parameter subgroups of $G, \phi$ is isolated (see (b) above).

(d) $Z$ is finite Conditions (i) and (ii) are equivalent. The argument in (c), shows that Condition (ii) is equivalent to Condition (i) holding for $p \phi$. Since $Z$ is finite, Condition (i) holding for $p \phi$ is equivalent to Condition (i) holding for ф. Q.E.D. (Theorem 4)

8. Theorem 5. Theorem 5 , which concerns semisimple Lie groups, shows the sufficiency of Condition (c) of Theorem A (Introduction). The weaker sufficient condition on $\phi$ is that $\phi^{*}$ be ergodic. Such $\phi$ are completely characterized by Moore's ergodicity theorem as follows $[\mathbf{2 0} ; \mathbf{5}$, p. 598; 34].

Let $G$ satisfy the conditions of Theorem 5 (below). In this restricted context the definitions and assumptions involved in Moore's theorem reduce to the following. A lattice $\Gamma$ in $G$ is reducible if and only if there exist connected normal subgroups $G_{1}$ and $G_{2}$ such that $G=G_{1} \times G_{2}$ and $\left(\Gamma \cap G_{1}\right) \times\left(\Gamma \cap G_{2}\right)$ is a subgroup of finite index in $\Gamma$. A lattice $\Gamma$ in $G$ is irreducible if and only if it is not reducible. Let $\Gamma$ be any lattice in $G$. Then $G=G_{1} \times \cdots \times G_{n}$, where, for $i=1, \ldots, n, G_{i}$ is a connected normal subgroup of $G, \Gamma_{i} \equiv \Gamma \cap G_{i}$ is an irreducible lattice in $G_{i}$, and $\Gamma_{1} \times \cdots \times \Gamma_{n}$ is a subgroup of finite index in $\Gamma[26, p$. 86]. Let $\phi$ be a one-parameter subgroup of $G$, and let $\phi_{i}$ be its projection on $G_{i}$. Then, by Moore's theorem, $\phi^{*}$ on $G / \Gamma$ is ergodic if and only if, for all $i, \phi_{i}$ has noncompact closure in $G_{i}$. It follows that if $\phi^{*}$ on $G / \Gamma$ is ergodic, then $\phi^{*} \times \phi^{*}$ on $G / \Gamma \times G / \Gamma$ is also ergodic and thus that $\phi^{*}$ on $G / \Gamma$ is weakly mixing $[\mathbf{2 4}$, p. 65$]$. It is also clear that Condition (2) of Theorem 5 is weaker than Condition (1) (Example 13, §10).

THEOREM 5. Let $\Gamma$ and $\Gamma^{\prime}$ be lattices in $G$ and $G^{\prime}$ respectively, where $G$ and $G^{\prime}$ are connected, centerless, semisimple Lie groups with no compact direct factors and with no direct factor $H$ such that both $H$ is isomorphic to $\operatorname{PSL}(2, \mathbf{R})$ and $\Gamma H$ is a closed subgroup of $G$. Let $\phi$ and $\psi$ be one-parameter subgroups of $G$ and $G^{\prime}$ respectively. It follows that if Condition (1) or Condition (2) hold for $\phi$, then $\phi^{*}$ and $\psi^{*}$ are topologically equivalent if and only if they are affinely equivalent. 

$\phi$.

CONDITION (1). There is no compact connected subgroup in the centralizer of

Condition (2). $\phi^{*}$ is ergodic.

ProOf. Lemma 1 (below), allows us to consider $G=G^{\prime}$ and $\Gamma=\Gamma^{\prime}$.

LEMMA 1. Let $G$ and $G^{\prime}$ be connected, centerless, semisimple Lie groups, with lattices $\Gamma$ and $\Gamma^{\prime}$ and identity elements $e$ and $e^{\prime}$ respectively. Then any homeomorphism $f:(G / \Gamma, e \Gamma) \rightarrow\left(G^{\prime} / \Gamma^{\prime}, e^{\prime} \Gamma^{\prime}\right)$ lifts to a homeomorphism $\tilde{f}:(G, e) \rightarrow\left(G^{\prime}, e^{\prime}\right)$.

Note. To see that the conclusion of the lemma does not hold for any Lie group and lattice, consider the following example. $G=G^{\prime}=\mathbf{R} \times S^{1}, \Gamma=\Gamma^{\prime}=\mathbf{Z} \times e$, $G / \Gamma=S^{1} \times S^{1}$, and $f: S^{1} \times S^{1} \rightarrow S^{1} \times S^{1}$ such that $f(g, h)=(h, g)$ for all $g$ and $h$ in $S^{1}$.

ProOF OF Lemma 1. Let $p: G \rightarrow G / \Gamma$ and $p^{\prime}: G^{\prime} \rightarrow G^{\prime} / \Gamma^{\prime}$ be the canonical projections. By covering space theory, it is enough to show that $f_{*} p_{*} \pi_{1}(G, e) \subset$ $p_{*}^{\prime} \pi_{1}\left(G^{\prime}, e^{\prime}\right)\left[18\right.$, p. 156]. Therefore, it is enough to show that $p_{*} \pi_{1}(G, e)$ and $p_{*}^{\prime} \pi_{1}\left(G^{\prime}, e^{\prime}\right)$ are the centers of $\pi_{1}(G / \Gamma, e \Gamma)$ and $\pi_{1}\left(G^{\prime} / \Gamma^{\prime}, e^{\prime} \Gamma^{\prime}\right)$ respectively.

Consider the following commutative diagram of sequences where the horizontal rows are exact. $q: \tilde{G} \rightarrow G$ is the universal covering homomorphism, $Z(\tilde{G})$ is the center of $G$, and $\Delta \equiv q^{-1}(\Gamma)[30$, p. $62 ; \mathbf{1 8}$, p. 158].

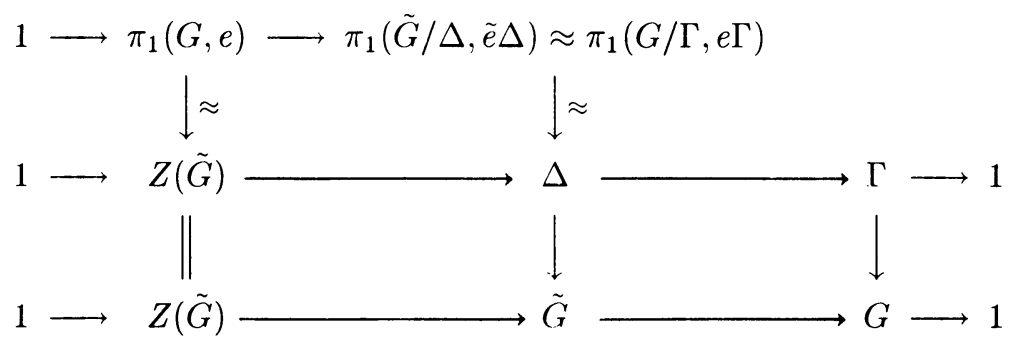

Since $G$ is centerless, the Borel density theorem implies that $\Gamma$ is centerless [26, pp. 84-87]. Therefore $Z(\tilde{G})$ is the center of $\Delta$, which implies that $p_{*} \pi_{1}(G, e)$ is the center of $\pi_{1}(G / \Gamma, e \Gamma)$.

The same argument shows that $p_{*}^{\prime} \pi_{1}\left(G^{\prime}, e^{\prime}\right)$ is the center of $\pi_{1}\left(G^{\prime} \Gamma^{\prime}, e^{\prime} \Gamma^{\prime}\right)$. Q.E.D. (Lemma 1)

Since $\phi^{*}$ and $\psi^{*}$ are topologically equivalent, $G / \Gamma$ and $G^{\prime} / \Gamma^{\prime}$ are homeomorphic by a map $f$, which we can take, after translation, to be basepoint preserving. By Lemma $1, f$ lifts to $\tilde{f}:(G, e) \rightarrow\left(G^{\prime}, e^{\prime}\right) . \tilde{f}$ restricted to $\Gamma$ is an isomorphism from $\Gamma$ to $\Gamma^{\prime}$. Since the conditions on $G, G^{\prime}, \Gamma$, and $\Gamma^{\prime}$ are precisely the conditions to which the Margulis-Prasad version of the Mostow rigidity theorem applies, $\tilde{f}_{\left.\right|_{\Gamma}}$ extends to an isomorphism $A: G \rightarrow G^{\prime}[\mathbf{2 2}$, p. $158, \mathbf{1 7}, \mathbf{2 5}, \mathbf{3 4}$, p. 85].

Therefore, we can consider $G=G^{\prime}$ and $\Gamma=\Gamma^{\prime}$ (Lemma 1 of $\S 2$ ).

If Condition (1) holds, we verify that the conditions of Theorem 1(c) hold. By Lemma $1,(G, G / \Gamma)$ has the homeomorphism lifting property. By the Mostow rigidity theorem, $(G, \Gamma)$ has the automorphism extension property. By Theorem $4, \phi$ is isolated. Therefore, by Theorem $1(\mathrm{c})$, it follows that Theorem 5 is proved wherever Condition (1) holds.

For the remainder of this proof, we assume that $\phi^{*}$ is ergodic (Condition (2)), and $G$ is a subgroup of $\operatorname{SL}(n, \mathbf{R})$ by means of the adjoint representation (see proof of Theorem 4). Let $\mu$ be the left invariant Borel measure on $G / \Gamma$. 
As in the proof of Theorem 1, we can assume that the topological equivalence of $\phi^{*}$ and $\psi^{*}$ has a lift $\tilde{f}:(G, e) \rightarrow(G, e)$ such that $\tilde{f}_{\mid \Gamma}=$ identity on $\Gamma$, and that, therefore, $\phi$ is recurrently approached by $\psi$. It follows, by Theorem 3 , that we can assume that $\psi$ has been reparameterized so that, for all $t, \phi(t)=E_{1}(t) \chi(t)$ and $\psi(t)=E_{2}(t) \chi(t)$, where $E_{1}, E_{2}$, and $\chi$ are subgroups of $G$ having the properties referred to in Theorem 3 .

LEMMA 2. There exists a subset $S$ of $G / \Gamma$ with $\mu(S)=\mu(G / \Gamma)$, such that if $x \Gamma \in S$, then $\tilde{f}(x) x^{-1} \in N_{G}(\chi)$, the connected component of the normalizer in $G$ of $\chi$.

Proof OF LemMa 2. Let $S$ be the subset of $G / \Gamma$ such that if $x \Gamma \in S$, then there exists a compact set $D \subset G / \Gamma$ and a sequence

$$
\left\{t_{k} \mid k \in \mathbf{Z}, t_{k} \in \mathbf{R}, \operatorname{sign}(k)=\operatorname{sign}\left(t_{k}\right), t_{k} \rightarrow \pm \infty \text { as } k \rightarrow \pm \infty\right\},
$$

such that, for all $k \in \mathbf{Z}, \phi^{*}\left(t_{k}, x \Gamma\right) \equiv \phi\left(t_{k}\right) x \Gamma \in D$. By the Poincaré recurrence theorem $\left[\mathbf{7}\right.$, p. 8], since $\phi^{*}$ is a measure preserving flow, $\mu(S)=\mu(G / \Gamma)$.

Let $x \Gamma \in S$. Let $g \equiv \tilde{f}(x) x^{-1}$. Note that $g$ is independent of the choice of coset representative.

$$
\left\{\tilde{f}\left(\phi\left(t_{k}\right) x\right)\left(\phi\left(t_{k}\right) x\right)^{-1}: k \in \mathbf{Z}\right\} \subset\left\{\tilde{f}(x) x^{-1}: x \Gamma \in D\right\},
$$

which is compact by Lemma 2 of Theorem 1 . Therefore $\left\{\tilde{f}\left(\phi\left(t_{k}\right) x\right)\left(\phi\left(t_{k}\right) x\right)^{-1}: k \in\right.$ Z h has compact closure. Since $f$ is a topological equivalence of $\phi^{*}$ and $\psi^{*}$, there exists a sequence

$$
\left\{s_{k} \mid k \in \mathbf{Z}, s_{k} \in \mathbf{R}, \operatorname{sign}(k)=\operatorname{sign}\left(s_{k}\right), s_{k} \rightarrow \pm \infty \text { as } k \rightarrow \pm \infty\right\},
$$

such that $f\left(\phi\left(t_{k}\right) x \Gamma\right)=\psi\left(s_{k}\right) f(x \Gamma)$, for all $k \in \mathbf{Z}$. Lifting to $G$, we obtain $\tilde{f}\left(\phi\left(t_{k}\right) x\right)=\psi\left(s_{k}\right) f(x)$, for all $k \in \mathbf{Z}$. Therefore, the set $\left\{\psi\left(s_{k}\right) g \phi\left(-t_{k}\right): k \in \mathbf{Z}\right\}$, which equals the set $\left\{\psi\left(s_{k}\right) \tilde{f}(x) x^{-1} \phi\left(-t_{k}\right): k \in \mathbf{Z}\right\}$, has compact closure. Therefore $\left\{g^{-1} \psi\left(s_{k}\right) g \phi\left(-t_{k}\right): k \in \mathbf{Z}\right\}$ has compact closure, and thus, by definition, the one-parameter subgroup $g^{-1} \psi g$ is recurrently approached by $\phi$. By Theorem 3 , there exists $\beta>0$ and a one-parameter subgroup $E_{3}$ with compact closure, such that, for all $t \in \mathbf{R}, g^{-1} \psi(\beta t) g=E_{3}(t) \chi(t)$. Therefore $g^{-1} E_{2}(\beta t) g g^{-1} \chi(\beta t) g$ equals $E_{3}(t) \chi(t)$. By the uniqueness of the decomposition of Theorem 3 , we obtain, for all $t \in \mathbf{R}$, that $g^{-1} \chi(\beta t) g=\chi(t)$. Therefore $g$ is in $N_{G}(\chi)$. Q.E.D. (Lemma 2)

LEMMA 3. Let $f: G / \Gamma \rightarrow G / \Gamma$ be a topological equivalence of the induced flows $\phi^{*}$ and $\psi^{*}$, such that the lift $\tilde{f}: G \rightarrow G$ has $\tilde{f}_{\mid \Gamma}=$ identity on $\Gamma$. Then there exists a one-parameter subgroup $\psi^{\prime}$ of $G$, a homeomorphism $f^{\prime}: G / \Gamma \rightarrow G / \Gamma$ with lift $\tilde{f}^{\prime}: G \rightarrow G$, and a set $S \subset G / \Gamma$ invariant under $\phi^{*}$ with $\mu(S)=\mu(G / \Gamma)$, such that

(i) $\psi$ is conjugate to $\psi^{\prime}$, and hence $\psi^{*}$ and $\psi^{\prime *}$ are affinely equivalent,

(ii) $\tilde{f}^{\prime}(x \gamma)=\tilde{f}^{\prime}(x) \gamma$, for all $x \in G$ and $\gamma \in \Gamma$,

(iii) $f^{\prime}$ is a topological equivalence of $\phi^{*}$ and $\psi^{\prime *}$, and

(iv) for almost all $x \Gamma$ in $S, \tilde{f}^{\prime}(x) x^{-1} \in K_{2}^{\prime} K_{1} \chi$, where, for some $\beta>0, \phi(t)=$ $E_{1}(t) \chi(t)$ and $\psi^{\prime}(t)=E_{2}^{\prime}(t) \chi(\beta t)$ are decompositions as in Theorem 3 , and $K_{2}^{\prime}$ and $K_{1}$ are the closures of $E_{2}^{\prime}$ and $E_{1}$.

ProOF OF LEMMA 3. Let $S \subset G / \Gamma$ be as in Lemma 2. Let $g: G \rightarrow G$, $g: x \mapsto \tilde{f}(x) x^{-1}$. Note that $g(x \gamma)=g(x)$, for all $x \in G$ and $\gamma \in \Gamma$. Let $K_{2}$ 
be the closure of $E_{2}$. By Lemma $2, g$ induces a map $S \rightarrow K_{2} \backslash N_{G}(\chi) / \chi K_{1}$, $x \Gamma \mapsto K_{2} \backslash g(x) / \chi K_{1}$. First we show that this map is $\phi^{*}$ invariant.

For all $t$, there exists $s$ and $s^{\prime}$ such that

$$
\begin{aligned}
g(\phi(t) x) & =\tilde{f}(\phi(t) x)(\phi(t) x)^{-1} \\
& =\psi(s) \tilde{f}(x) x^{-1} \phi(-t) \quad(f \text { is a topological equivalence) } \\
& =E_{2}(s) \chi(s) g(x) E_{1}(-t) \chi(-t) \\
& =E_{2}(s) g(x) \chi\left(s^{\prime}\right) E_{1}(-t) \chi(-t) \quad(\text { Lemma } 2) \\
& =E_{2}(s) g(x) \chi\left(s^{\prime}-t\right) E_{1}(-t)
\end{aligned}
$$

Therefore

$$
K_{2} \backslash g(\phi(t) x) / \chi K_{1}=K_{2} \backslash g(x) / \chi K_{1},
$$

which shows the $\phi^{*}$ invariance of the map $x \Gamma \mapsto K_{2} \backslash g(x) / \chi K_{1}$.

Since $\phi^{*}$ is ergodic, the only a.e. invariant functions are a.e. constants. Therefore $x \Gamma \mapsto K_{2} \backslash g(x) / \chi K_{1}$ is a.e. constant. Thus there exists $h \in N_{G}(\chi)$ such that a.e. in $S, K_{2} \backslash g(x) / \chi K_{1}$ equals $K_{2} \backslash h / \chi K_{1}$. Therefore for almost all $x \Gamma$ in $S, g(x)$ is a member of the set $K_{2} h \chi K_{1}$, and $h^{-1} g(x) \in h^{-1} K_{2} h \chi K_{1}$. Since $h \in N_{G}(\chi)$, there exists $\beta>0$ such that, for all $t, h^{-1} \chi(t) h=\chi(\beta t)$. It is easy to see that $K_{2}^{\prime}$ centralizes $\chi$, where $K_{2}^{\prime} \equiv h^{-1} K_{2} h$. Therefore for almost all $x \Gamma$ in $S$, $h^{-1} g(x) \in K_{2}^{\prime} K_{1} \chi$.

Lemma 3 follows by making $f^{\prime} \equiv \overline{h^{\prime}} \circ f, \tilde{f}^{\prime}(x) \equiv h^{-1} \tilde{f}(x)$ for all $x \in G$, and, for all $t, \psi^{\prime}(t) \equiv h^{-1} \psi(t) h$ and $E_{2}^{\prime}(t) \equiv h^{-1} E_{2}(t) h$. Q.E.D. (Lemma 3)

LEMMA 4. Let $\phi(t)=E_{1}(t) \chi(t)$ and $\psi(t)=E_{2}(t) \chi(\beta t), \beta>0$, be oneparameter subgroups of $G$ with a decomposition as in Theorem 3. Let $f: G / \Gamma \rightarrow$ $G / \Gamma$ be a topological equivalence of $\phi^{*}$ and $\psi^{*}$ with lift $\tilde{f}: G \rightarrow G$ such that $\tilde{f}(x \gamma)=$ $\tilde{f}(x) \gamma$ for all $x \in G$ and $\gamma \in \Gamma$. Let $S$ be a subset of $G / \Gamma$ with $\mu(S)=\mu(G / \Gamma)$, which is invariant under $\phi^{*}$ and such that, for all $x \Gamma \in S, g(x) \in K_{2} K_{1} \chi$, where $g(x) \equiv \tilde{f}(x) x^{-1}$. Then there exists a measurable equivalence (not necessarily timepreserving), $f: S \rightarrow S$ of $\phi^{*}$ and $\psi^{*}$ with lift $\tilde{f}^{\prime}$ such that $\tilde{f}^{\prime}(x \gamma)=\tilde{f}^{\prime}(x) \gamma$ and such that $\tilde{f}^{\prime}(x) x^{-1} \in K_{2} K_{1}$.

PROOF OF LEMMA 4. The idea behind the proof is to slide $f$ along the flow lines of $\phi^{*}$ to get an equivalence $f^{\prime}$ such that $\tilde{f}^{\prime}(x) x^{-1} \in K_{2} K_{1}$.

First we note that if, for some $t \in \mathbf{R}, k_{2} \in K_{2}$, and $k_{1} \in K_{1}, \chi(t)=k_{2} k_{1}$, then $\left\{k_{2}^{-n} \chi(n t): n \in \mathbf{Z}\right\}=\left\{k_{1}^{n}: n \in \mathbf{Z}\right\}$, which has compact closure. Therefore $t=0$ (see Theorem 3). Thus $\chi \cap K_{2} K_{1}=e$.

It follows that $K_{2} K_{1} \times R \rightarrow K_{2} K_{1} \chi,\left(k_{2} k_{1}, t\right) \mapsto k_{2} k_{1} \chi(t)$ is a homeomorphism. For $x \Gamma \in S$, let $k_{2}(x), k_{1}(x), T(x)$ correspond to $g(x)$ by means of this homeomorphism. $T(x \gamma)=T(x)$, since $g(x \gamma)=\tilde{f}(x \gamma)(x \gamma)^{-1}=\tilde{f}(x) x^{-1}=g(x) . T$ is measurable on $S$.

Define $f^{\prime}: S \rightarrow S$ and its lift $\tilde{f}^{\prime}$, by

$$
\tilde{f}^{\prime}(x) \equiv \psi((-T(x)) / \beta) \tilde{f}(x) \quad \text { and } \quad f^{\prime}(x \Gamma) \equiv \psi((-T(x)) / \beta) \tilde{f}(x) \Gamma .
$$

Then

$$
\begin{aligned}
\tilde{f}^{\prime}(x) x^{-1} & =\psi((-T(x)) / \beta) \tilde{f}(x) x^{-1} \\
& =E_{2}((-T(x)) / \beta) \chi(-T(x)) k_{2}(x) k_{1}(x) \chi(T(x)) \\
& =E_{2}((-T(x)) / \beta) k_{2}(x) k_{1}(x) \in K_{2} K_{1} .
\end{aligned}
$$

Q.E.D. (Lemma 4) 
LEMMA 5. Let $\phi$ and $\psi$ and $S$ be as in Lemma 4. Let $f: S \rightarrow S$ be a measurable equivalence (not necessarily time-preserving) of $\phi^{*}$ and $\psi^{*}$ with lift $\tilde{f}$ such that, for all $x \Gamma \in S$ and $\gamma \in \Gamma, \tilde{f}(x \gamma)=\tilde{f}(x) \gamma$ and $g(x) \in K_{2} K_{1}$, where $g(x) \equiv \tilde{f}(x) x^{-1}$. Let $\phi^{*}$ be ergodic. Then $\phi^{*}$ and $\psi^{*}$ are affinely equivalent by the identity.

Proof of LemMa 5. For $x \Gamma \in S$, for all $t \in \mathbf{R}$ there exists $s \in \mathbf{R}$, such that

$$
\begin{aligned}
g(\phi(t) x) & =\tilde{f}(\phi(t) x) x^{-1} \phi(-t)=\psi(s) \tilde{f}(x) x^{-1} \phi(-t) \\
& =\psi(s) g(x) \phi(-t)=E_{2}(s) \chi(\beta s) g(x) E_{1}(-t) \chi(-t) \\
& =E_{2}(s) g(x) E_{1}(-t) \chi(\beta s-t) \quad\left(g(x) \in K_{2} K_{1}\right) \\
& =E_{2}(t / \beta) g(x) E_{1}(-t) \quad\left(\text { range of } g \text { is } K_{2} K_{1} \text { implies } \beta s-t=0\right) .
\end{aligned}
$$

Therefore, $x \Gamma \mapsto g(x) K_{1}$ is a measurable conjugacy (many to one, but timepreserving), of $\phi^{*}$ onto the flow $\left(k_{2} k_{1} K_{1}, t\right) \mapsto E_{2}(t / \beta) k_{2} k_{1} K_{1}$ on the space $\left(K_{2} K_{1}\right) / K_{1}$. $\left(K_{2} K_{1}\right) / K_{1} \rightarrow K_{2} /\left(K_{1} \cap K_{2}\right), k_{2} k_{1} K_{1} \mapsto k_{2}\left(K_{1} \cap K_{2}\right)$ is a topological conjugacy of the flow $\left(k_{2} k_{1} K_{1}, t\right) \mapsto E_{2}(t / \beta) k_{2} k_{1} K_{1}$ and the flow $\left(k_{2}\left(K_{1} \cap K_{2}\right), t\right)$ $\mapsto\left(E_{2}(t / \beta)\left(K_{1} \cap K_{2}\right)\right)\left(k_{2}\left(K_{1} \cap K_{2}\right)\right)$, which is topologically conjugate to a oneparameter subgroup induced toral flow.

By the Moore ergodicity theorem (see beginning of $\S 8$ ), $\phi^{*}$ is weakly mixing.

For any weakly mixing flow the only eigenfunctions are constants. Now suppose that a weakly mixing flow is measurably conjugate (possibly many to one, but time-preserving) onto an induced toral flow. Since the conjugacy followed by a projection onto a factor of the torus is an eigenfunction, it follows that the toral flow is induced by the identity subgroup.

It follows that, for all $t, E_{2}(t / \beta)\left(K_{1} \cap K_{2}\right)=K_{1} \cap K_{2}$. Therefore, $E_{2}$ and its closure $K_{2}$ are contained in $K_{1}$.

It follows that $S \rightarrow K_{1}, x \Gamma \mapsto g(x)$, is a measurable conjugacy (many to one, but time-preserving) of $\phi_{\mid S}^{*}$ onto the flow on $K_{1}$ induced by the subgroup $t \mapsto$ $E_{2}(t / \beta) E_{1}(-t)$. By the same argument as above, since $\phi^{*}$ is weakly mixing, it follows that, for all $t, E_{2}(t / \beta) E_{1}(-t)=e$. Therefore, for all $t, E_{2}(t)=E_{1}(\beta t)$. Thus $\psi(t)=E_{2}(t) \chi(\beta t)=E_{1}(\beta t) \chi(\beta t)=\phi(\beta t)$. Q.E.D. (Lemma 5)

Lemma 2 through Lemma 5 complete the proof of the sufficiency of Condition (2). Q.E.D. (Theorem 5)

9. Proof of Theorem $\mathbf{A}$ and Theorem B. In this section, using the previous results, we prove Theorem $\mathrm{A}$ and Theorem $\mathrm{B}$ (Introduction).

PRoOF OF THEOREM A FOR CONDITION (a) ON NILPOTENT GROUPS. We can assume that $G=G^{\prime}$ and $\Gamma=\Gamma^{\prime}$, since if $G / \Gamma$ and $G^{\prime} / \Gamma^{\prime}$ are homeomorphic, they are homeomorphic by an affine map $\bar{A}: G / \Gamma \rightarrow G^{\prime} / \Gamma^{\prime}$. Furthermore, $(G, \Gamma)$ has the automorphism extension property ( $\S 2$ (Lemma 1) and $[\mathbf{1 5}$, Theorem $5, \mathrm{p}$. 292]).

We verify that the other conditions of Theorem $1(\mathrm{c})$ hold. $(G, G / \Gamma)$ has the homeomorphism lifting property, since $G$ is simply connected. By Theorem $2, \phi$ is isolated. Q.E.D.

Proof of TheOREM A FOR CONDITION (b) ON SOlvable Groups. By the condition that the eigenvalues are real, we can assume that $G=G^{\prime}$ and $\Gamma=\Gamma^{\prime}$. Because, if $G / \Gamma$ and $G^{\prime} / \Gamma^{\prime}$ are homeomorphic, they are homeomorphic by an affine 
map. Furthermore, $(G, \Gamma)$ has the automorphism extension property ( $§ 2$ (Lemma 1), Gorbacevic [10], Mosak and Moskowitz [21], Saito [29, p. 166]; see §10(f)).

We verify that the other conditions of Theorem $1(\mathrm{c})$ hold. $(G, G / \Gamma)$ has the homeomorphism lifting property, since $G$ is simply connected. Since for all $X$ in $L(G)$, no eigenvalue of $\operatorname{ad}(X)$ is pure imaginary, it follows that the exponential map is a diffeomorphism (Saito [29, and 6, p. 400]). Therefore, by Theorem 2, $\phi$ is isolated. Q.E.D.

Theorem A for Condition (c) on semisimple groups is Theorem 5. Q.E.D. (Theorem A)

PROOF OF THEOREM B. Theorem B(a), which concerns nilpotent and solvable groups, is Theorem 2. Theorem $\mathrm{B}(\mathrm{b})$, which concerns semisimple groups, is Theorem 4. Q.E.D. (Theorem B)

10. Examples, comments, and questions. In this section we comment on, ask further questions raised by, illustrate, and show the limits of our theorems.

(a) EXAMPLE 1. A nilpotent example of Theorem A is the well-known Heisenberg group, the group of $(3,3)$ matrices with 0 below the diagonal, 1 on the diagonal, and any real numbers above the diagonal. Take the subgroup with integral entries as the lattice $\Gamma$.

(b) EXAMPLE 2. Nonnilpotent solvable examples of Theorem A can be obtained by starting with a matrix in $\mathrm{SL}(n, \mathbf{Z})$ which is semisimple with all its eigenvalues real. Then there exists an action $\sigma: R \rightarrow \mathrm{SL}(n, \mathbf{R})$ of $\mathbf{R}$ on $\mathbf{R}^{n}$ such that $\sigma(1)$ is the matrix we started with. Using the action $\sigma$, let $G=\mathbf{R}^{n} \rtimes \mathbf{R}$, and using the action $\sigma_{\mid \Gamma}$, let $\Gamma=\mathbf{Z}^{n} \rtimes \mathbf{Z}$.

(c) EXAMPLE 3. The condition for solvable groups (Theorem A, Condition (b)) is not necessary. The following is an example of a connected, simply connected, solvable Lie group for which an eigenvalue of $\operatorname{ad}(X)$ is pure imaginary. But still topologically equivalent flows $\phi_{1}^{*}$ and $\phi_{2}^{*}$ are affinely equivalent.

Let $\sigma(t)$ be the counterclockwise rotation of $\mathbf{R}^{2}$ by $2 \pi t$. Let $S_{2}$ be the semidirect product $\mathbf{R}^{2} \rtimes \mathbf{R}$, where $\sigma$ is the action of $\mathbf{R}$ on $\mathbf{R}^{2}$. Let $\Gamma_{2}$ be the subgroup $\mathbf{Z}^{2} \rtimes \mathbf{Z}$, which is isomorphic to $\mathbf{Z}^{3}$ since $\sigma(1)$ is the identity. Then $S_{2}$ is a connected, simply connected, solvable Lie group. $S_{2} / \Gamma_{2}$ is compact. $\mathbf{R}^{2}$ is the commutator of $S_{2}$. There is an $X$ in $L\left(S_{2}\right)$ such that $\operatorname{ad}(X)$ has eigenvalue $2 \pi i(X=1 \in$ $L(\mathbf{R})=\mathbf{R})$. The exponential map is not a diffeomorphism since in fact all oneparameter subgroups not contained in $\mathbf{R}^{2}$ intersect [3, pp. 18-21, 31-38]. $\left(S_{2}, \Gamma_{2}\right)$ does not have the automorphism extension property, since, for example, the map $(x, y, z) \mapsto(A(x, y), z)$ is an automorphism of $\Gamma_{2}$ for any $A$ in $\mathrm{SL}(2, \mathbf{Z})$, but it is an automorphism of $S_{2}$ if and only if, for all $t, A$ commutes with $\sigma(t)$. But $A \sigma(1 / 4) \neq \sigma(1 / 4) A$, where $A=\left(\begin{array}{ll}1 & 1 \\ 0 & 1\end{array}\right)$.

Any one-parameter subgroup contained in the subgroup $\mathbf{R}^{2}$ is conjugate to a subgroup of the form $t \mapsto(t x, 0,0), x>0$. Any one-parameter subgroup not contained in $\mathbf{R}^{2}$ is conjugate to a subgroup of the form $t \mapsto(0,0, t z)$. The map $(x, y, z) \mapsto(y, x,-z)$, which is an automorphism of $S_{2}$ and of $\Gamma_{2}$, takes a subgroup of the form $t \mapsto(0,0, t z)$ where $z$ is negative to a subgroup of the form $t \mapsto(0,0, t z)$ where $z$ is positive. Therefore it follows that the flows $\phi_{1}^{*}$ and $\phi_{2}^{*}$ are topologically equivalent by an affine map if both the subgroups $\phi_{1}$ and $\phi_{2}$ are contained in $\mathbf{R}^{2}$ or if neither of the subgroups $\phi_{1}$ and $\phi_{2}$ are contained in $\mathbf{R}^{2}$ (§2 (Lemma 1)). 
However, if $\phi_{1}$ is contained in $\mathbf{R}^{2}$ and $\phi_{2}$ is not contained in $\mathbf{R}^{2}$, then the flows $\phi_{1}^{*}$ and $\phi_{2}^{*}$ are not topologically equivalent. To see this consider $\phi_{1}: t \mapsto(t, t y, 0)$ where $y$ is irrational, and $\phi_{2}: t \mapsto(0,0, t)$. All orbits in the flow $\phi_{2}^{*}$ are periodic, but in the flow $\phi_{1}^{*}$ the orbit of $(0,0,0) \Gamma_{2}$ is not periodic.

(d) EXAMPLE 4. In the solvable case, sometimes when Condition (b) of Theorem A fails, the conclusion fails. That is there are topologically equivalent flows which are not affinely equivalent. The following example is in $[\mathbf{3}, \mathrm{p} .33]$.

Let $G=S_{2}$ and $\Gamma=\Gamma_{2}$ from Example 3 above. Let $G^{\prime}=\mathbf{R}^{3}$ and $\Gamma^{\prime}=\mathbf{Z}^{3}$. Let $\phi_{1}: t \mapsto(0,0,-t)$ be a subgroup of $S_{2}$, and $\phi_{2}: t \mapsto(0,0, t)$ a subgroup of $\mathbf{R}^{3}$. $f:(x, y, z) \mapsto(-\sigma(-z)(x, y),-z)$ is a diffeomorphism from $S_{2}$ to $\mathbf{R}^{3}$ which induces a diffeomorphism $\bar{f}$ from $S_{2} / \Gamma_{2}$ to $\mathbf{R}^{3} / \mathbf{Z}^{3}$. $\bar{f}$ is a topological equivalence, and in fact a topological conjugacy, of $\phi_{1}^{*}$ and $\phi_{2}^{*}$, but $\phi_{1}^{*}$ and $\phi_{2}^{*}$ are not affinely equivalent since $S_{2}$ and $\mathbf{R}^{3}$ are not isomorphic.

(e) EXAMPLE 5. In the solvable case, sometimes when Condition (b) of Theorem A fails, the conclusion fails even when $G=G^{\prime}$ and $\Gamma=\Gamma^{\prime}$.

Let $G=G^{\prime}=S_{2} \times \mathbf{R}^{3}$ and $\Gamma=\Gamma^{\prime}=\Gamma_{2} \times \mathbf{Z}^{3}$. The subgroups $\phi_{1}$ of $S_{2}$ and $\phi_{2}$ of $\mathbf{R}^{3}$ referred to in Example 4 can be considered as subgroups of $G$. The map $f$ of Example 2 can be considered as a diffeomorphism of $\mathbf{R}^{3}$ with $S_{2}$ as well. $f=f^{-1}$. Then $F(g, h) \equiv(f(h), f(g))$ is a diffeomorphism of $G$ with $G$ which induces a diffeomorphism $\bar{F}$ of $G / \Gamma$ with $G / \Gamma . \bar{F}$ is a topological equivalence, and in fact a topological conjugacy, of $\phi_{1}^{*}$ and $\phi_{2}^{*}$.

Suppose $\phi_{1}^{*}$ and $\phi_{2}^{*}$ are affinely equivalent. Then there exists $a \in G, s>0$, and an automorphism $A$ of $G$ which extends an automorphism of $\Gamma$, such that $\left(A \phi_{1}\right)(t)=\left(a \phi_{2} a^{-1}\right)(s t)\left(\S 2\right.$, Lemma 1). Since $\phi_{2}$ is a subgroup of $\mathbf{R}^{3}, a \phi_{2} a^{-1}=$ $\phi_{2}$. Since $A(\Gamma)=\Gamma, s$ must equal 1 . So $A \phi_{1}=\phi_{2}$.

Let $A^{\prime}: \mathbf{R}^{2} \rightarrow \mathbf{R}^{2}$ be the isomorphism gotten by restricting $A$ to the commutator $[G, G]$, which is the normal subgroup $\mathbf{R}^{2}$ of $S_{2}$. It must be the case that

$$
A:((x, y,-t),(0,0,0)) \rightarrow\left(\left(A^{\prime}(x, y), 0\right),(0,0, t)\right) .
$$

But then there exists $g_{1}=\left(\left(x_{1}, y_{1},-t_{1}\right),(0,0,0)\right)$ and $g_{2}=\left(\left(x_{2}, y_{2},-t_{2}\right),(0,0,0)\right)$ such that $A\left(g_{1} g_{2}\right) \neq A\left(g_{1}\right) A\left(g_{2}\right)$. This contradiction shows that the flows $\phi_{1}^{*}$ and $\phi_{2}^{*}$ are not affinely equivalent.

(f) Automorphism extension for solvable groups. In Condition (b) of Theorem $\mathrm{A}$, we require that $\operatorname{ad}(X)$ have only real eigenvalues in order to insure that $(G, \Gamma)$ has the automorphism extension property, which is required in Theorem 1(c). The following example shows that not every $(G, \Gamma)$ has the automorphism extension property, where $\Gamma$ is a lattice in $G$ and $G$ is a connected, simply connected, solvable Lie group such that exp: $L(G) \rightarrow G$ is a diffeomorphism (Milovanov [19]).

EXAMPLE 6 . Let $R_{\theta}$ be clockwise rotation of $\mathbf{R}^{2}$ by $\theta$. Let $\sigma(t)$ be in $\operatorname{SL}(4, \mathbf{R})$ where

$$
\sigma(t):\left(x_{1}, x_{2}, x_{3}, x_{4}\right) \mapsto\left(e^{k t} R_{2 \pi t}\left(x_{1}, x_{2}\right), e^{-k t} R_{2 \pi t}\left(x_{3}, x_{4}\right)\right),
$$

and $e^{k}+e^{-k}=m$ for some integer $m>2$. Let $G \equiv \mathbf{R}^{4} \rtimes \mathbf{R}$, where $\mathbf{R}$ acts on $\mathbf{R}^{4}$ by $\sigma$. Let $X_{1}, X_{2}, X_{3}$, and $X_{4}$ be the standard basis of $\mathbf{R}^{4}$. Let $c \equiv$ $e^{k}-e^{-k}$. Let $Y_{1} \equiv-e^{-k} X_{1}+e^{k} c^{-1} X_{3}, Y_{2} \equiv-e^{-k} X_{2}+e^{k} c^{-1} X_{4}, Y_{3} \equiv-X_{1}+$ $c^{-1} X_{3}$, and $Y_{4} \equiv-X_{2}+c^{-1} X_{4}$. The $\left\{Y_{i}\right\}$ is a basis and it generates $\Gamma_{u}$, a uniform discrete subgroup of $\mathbf{R}^{4}$. Since $\sigma(1)\left(Y_{1}\right)=Y_{3}, \sigma(1)\left(Y_{2}\right)=Y_{4}, \sigma(1)\left(Y_{3}\right)=$ $-Y_{1}+m Y_{3}$, and $\sigma(1)\left(Y_{4}\right)=-Y_{2}+m Y_{4}$, it follows that $\sigma(1)$ is an automorphism 
of $\Gamma_{u}$. Therefore $\Gamma \equiv \Gamma_{u} \rtimes \mathbf{Z}$ is a uniform discrete subgroup of $G$. For any $A \in \mathrm{SL}(2, \mathbf{Z}), \alpha:\left(x_{1}, x_{2}, x_{3}, x_{4}\right) \mapsto\left(A\left(x_{1}, x_{2}\right), A\left(x_{3}, x_{4}\right)\right)$ is an automorphism of $\Gamma_{u}$ which commutes with $\sigma(1)$. Therefore $\psi:(v, r) \mapsto(\alpha v, r)$, for all $(v, r)$ in $\Gamma_{u} \rtimes \mathbf{Z}$, is an automorphism of $\Gamma$. Any extension of $\psi$ to $G$ must be of the form $(v, r) \mapsto(\alpha v, r)$, for all $(v, r)$ in $G$. But if $A$ has been chosen to be $\left(\begin{array}{ll}1 & 1 \\ 0 & 1\end{array}\right)$, then the map $(v, r) \mapsto(\alpha v, r)$ is not an automorphism of $G$ since $\sigma(1 / 4) \alpha \neq \alpha \sigma(1 / 4)$.

Question. Which $(G, \Gamma)$ have the automorphism extension property, where $G$ is a connected, simply connected, solvable Lie group with $\exp : L(G) \rightarrow G$ a diffeomorphism, and $\Gamma$ is a lattice in $G$ ?

(g) Semisimple case. We first give examples of isolated and not isolated subgroups.

EXAMPLE 7. Let $G$ be a semisimple subgroup of $\operatorname{SL}(n, \mathbf{R})$. Let $\phi(t) \equiv e^{t X}$, for all $t$ in $\mathbf{R}$. If $X$ has $n$ distinct real eigenvalues, then $\phi$ is isolated.

ProOF. Consider $\phi$ as a subgroup of $\operatorname{SL}(n, \mathbf{R}) . \quad \phi$ does not have compact closure. If $[X, Y]=0$, then $Y$ is a diagonal matrix. Therefore, there is no compact, connected, subgroup in the centralizer of $\phi$. By Theorem $3, \phi$ is isolated as a subgroup of $\operatorname{SL}(n, \mathbf{R})$. Therefore, $\phi$ is isolated as a subgroup of $G$.

EXAMPLE 8. Every nontrivial unipotent subgroup of $\mathrm{SL}(3, \mathbf{R})$ is isolated.

PROOF. The proof is by contradiction.

Let $\phi: t \mapsto e^{t X}$, for all $t$ in $\mathbf{R}$, be a nontrivial unipotent one-parameter subgroup of $\operatorname{SL}(3, \mathbf{R})$ which is not isolated. Then, if $\phi$ is recurrently approached by $\psi$, it follows by Theorem 3 that $\psi=E \phi$, where, for all $t$ in $\mathbf{R}, E(t)=e^{t Y}, E(t) \phi(t)=$ $\phi(t) E(t), E$ has compact closure, and $X Y=Y X$. $X$ is nilpotent, and $Y$ must be conjugate to a matrix of the form

$$
\left(\begin{array}{ccc}
0 & -\theta & 0 \\
\theta & 0 & 0 \\
0 & 0 & 0
\end{array}\right)
$$

Thus $X$ and $Y$ are respectively the nilpotent and semisimple parts of the linear operator $X+Y$. Putting $X+Y$ in real canonical form $(\S 4,[13$, p. 126]), we see that $X$ must be 0 , which is a contradiction.

EXAMPLE 9. $\phi$ and $\phi^{\prime}$ are subgroups of $\operatorname{SL}(3, \mathbf{R})$, where for $t \in \mathbf{R}$,

$$
\phi(t)=\left(\begin{array}{ccc}
e^{t} & 0 & 0 \\
0 & e^{t} & 0 \\
0 & 0 & e^{-2 t}
\end{array}\right)
$$

and

$$
\phi^{\prime}(t)=\left(\begin{array}{ccc}
e^{t} \cos t & -e^{t} \sin t & 0 \\
e^{t} \sin t & e^{t} \cos t & 0 \\
0 & 0 & e^{-2 t}
\end{array}\right)
$$

Since $\phi$ is recurrently approached by $\phi^{\prime}$, neither $\phi$ nor $\phi^{\prime}$ is isolated. Observe that $\phi$ and $\phi^{\prime}$ have a compact subgroup isomorphic to the unit circle in their centralizer. 
EXAMPLE 10. Let $\phi$ and $\phi^{\prime}$ be subgroups of $\operatorname{SL}(4, \mathbf{R})$, where

$$
\phi(t)=\left(\begin{array}{cccc}
1 & 0 & 0 & 0 \\
t & 1 & 0 & 0 \\
0 & 0 & 1 & 0 \\
0 & 0 & 0 & 1
\end{array}\right)
$$

and

$$
\phi^{\prime}(t)=\left(\begin{array}{cccc}
1 & 0 & 0 & 0 \\
t & 1 & 0 & 0 \\
0 & 0 & \cos t & -\sin t \\
0 & 0 & \sin t & \cos t
\end{array}\right),
$$

for all $t$ in $\mathbf{R} . \phi$ is a unipotent subgroup which is recurrently approached in norm by $\phi^{\prime}$. Neither $\phi$ nor $\phi^{\prime}$ are isolated.

The following are examples to which Condition (c) of Theorem A applies.

EXAMPLE 11. Let $G, G^{\prime}, \Gamma$, and $\Gamma^{\prime}$ satisfy Condition (c) of Theorem A. In addition, let $G$ be a subgroup of $\mathrm{SL}(n, \mathbf{R})$ and let $\phi$ be chosen as in Example 7. To be more specific, we could let $G=\operatorname{PSL}(m, \mathbf{R})$ and $\Gamma=\operatorname{PSL}(m, \mathbf{Z})$, for any $m>2$ [26, Chapter 10]. Let $\phi^{\prime}$ be a subgroup of $G^{\prime}$. Then $\phi^{*}$ and $\phi^{\prime *}$ are topologically equivalent if and only if they are affinely equivalent.

EXAMPLE 12. Let $G=\operatorname{SL}(3, \mathbf{R})$, and let $\Gamma, G^{\prime}$, and $\Gamma^{\prime}$ satisfy Condition (c) of Theorem A. Let $\phi$ and $\phi^{\prime}$ be one-parameter subgroups of $G$ and $G^{\prime}$ respectively, with $\phi$ unipotent. Then $\phi^{*}$ and $\phi^{\prime *}$ are topologically equivalent if and only if they are affinely equivalent (Example 8).

EXAMPLE 13. Let $G=\operatorname{PSL}(n, \mathbf{R}), n>2$, and let $\Gamma, G^{\prime}$, and $\Gamma^{\prime}$ satisfy Condition (c) of Theorem A. Let $\phi$ and $\phi^{\prime}$ be one-parameter subgroups of $G$ and $G^{\prime}$ respectively, such that $\phi$ has noncompact closure (Example 9$)$. Then $\phi^{*}$ is ergodic (see beginning of $\S 8$ ). Therefore Condition $\mathrm{c}(2)$ is satisfied, and thus $\phi^{*}$ and $\phi^{\prime *}$ are topologically equivalent if and only if they are affinely equivalent.

(h) Topological equivalence of $G$-induced flows does not imply affine equivalence or topological conjugacy after constant reparameterization. Let $\Gamma$ and $\Gamma^{\prime}$ be uniform discrete subgroups of $G=G^{\prime}=\operatorname{PSL}(2, \mathbf{R})$. Then $G / \Gamma$ and $G / \Gamma^{\prime}$ are the unit tangent bundles to $S_{\Gamma}$ and $S_{\Gamma^{\prime}}$, compact surfaces with curvature -1 . Let

$$
\phi=\phi^{\prime}=q\left(\begin{array}{cc}
e^{t} & 0 \\
0 & e^{-t}
\end{array}\right) .
$$

$q: \operatorname{SL}(2, \mathbf{R}) \rightarrow \operatorname{PSL}(2, \mathbf{R})$ is the canonical projection. Then the flows $\phi^{*}$ and $\phi^{\prime *}$ on $G / \Gamma$ and $G / \Gamma^{\prime}$ are the geodesic flows of $S_{\Gamma}$ and $S_{\Gamma^{\prime}}[\mathbf{3}$, p. 26].

If $S_{\Gamma}$ is homeomorphic to $S_{\Gamma^{\prime}}$, then $\phi^{*}$ and $\phi^{\prime *}$ are topologically equivalent [11; 1, p. 26]. However, if there is no $a \in G$ such that $a \Gamma a^{-1}=\Gamma^{\prime}$, then there is no affine map from $G / \Gamma$ to $G / \Gamma^{\prime}$, and hence $\phi^{*}$ and $\phi^{\prime *}$ are not affinely equivalent. The existence of $\Gamma$ and $\Gamma^{\prime}$ such that $S_{\Gamma}$ and $S_{\Gamma^{\prime}}$ are homeomorphic compact surfaces, but $\Gamma$ and $\Gamma^{\prime}$ are not conjugate subgroups follows from the well-known fact that there exist compact surfaces of curvature -1 which are homeomorphic but not isometric.

Since a topological conjugacy of flows preserves the periods of closed orbits, a topological conjugacy of geodesic flows preserves the length of closed geodesics. 
Let the length spectra, $\operatorname{LS}(\Gamma)$ and $\operatorname{LS}\left(\Gamma^{\prime}\right)$, be the lengths with multiplicities of the closed geodesics on $S_{\Gamma}$ and $S_{\Gamma^{\prime}}$. There exist $\Gamma$ and $\Gamma^{\prime}$ such that $S_{\Gamma}$ and $S_{\Gamma^{\prime}}$ are homeomorphic but $\operatorname{LS}(\Gamma) \neq \operatorname{LS}\left(\Gamma^{\prime}\right)$, and, furthermore, there is no $\alpha>0$ such that $\alpha(\operatorname{LS}(\Gamma))=\operatorname{LS}\left(\Gamma^{\prime}\right)[\mathbf{3 3}]$. Therefore $\phi^{*}$ and $\phi^{\prime *}$ are topologically equivalent but not topologically conjugate. Furthermore, there is no constant reparameterization of $\phi^{\prime *}$ which would make the flows topologically conjugate.

\section{REFERENCES}

1. D. V. Anosov, Geodesic flows on closed Riemannian manifolds with negative curvature, Proc. Steklov Inst. Math. No. 90 (1967), Amer. Math. Soc., Providence, R.I., 1969.

2. L. Auslander, An exposition of the structure of solv-manifolds. I, II, Bull. Amer. Math. Soc. 79 (1973), 227-261, 262-285.

3. L. Auslander, L. Green, and F. Hahn, Flows on homogeneous spaces, Princeton Univ. Press, Princeton, N. J., 1963.

4. A. Beardon, The geometry of discrete groups, Springer-Verlag, New York, 1983.

5. J. Brezin and C. C. Moore, Flows on homogeneous spaces: a new look, Amer. J. Math. 103 (1981), 571613.

6. N. Bourbaki, Elements of mathematics, Lie groups and Lie algebras. I, Hermann, Paris, and Addison-Wesley, Reading, Mass., 1975.

7. I. P. Cornfeld, S. V. Fomin, and Ya. G. Sinai, Ergodic theory, Springer-Verlag, 1982.

8. S. G. Dani, On invariant measures, minimal sets, and a lemma of Margulis, Invent. Math. 51 (1979), $239-260$.

9. D. Fried, The geometry of cross sections to flows, Topology 21 (1982), 353-371.

10. V. V. Gorbacevic, Lattices in Lie groups of type $(E)$ and $(R)$, Vestnik Moskov. Univ. Ser. I Mat. Mekh. 30 (1975), 56-63; English transl., Moscow Univ. Math. Bull. 30 (1975), 98-104.

11. M. Gromov, Three remarks on geometric dynamics and fundamental groups, preprint.

12. S. Helgason, Differential geometry, Lie groups, and symmetric spaces, Academic Press, New York, 1978.

13. M. Hirsch and S. Smale, Differential equations, dynamical systems, and linear algebra, Academic Press, New York, 1974.

14. M. C. Irwin, Smooth dynamical systems, Academic Press, New York, 1980.

15. A. I. Malcev, On a class of homogeneous spaces, Izv. Akad. Nauk SSSR Ser. Mat. 13 (1949), 9-32; English transl., Amer. Math. Soc. Transl. (1) 9 (1962), 276-307.

16. B. Marcus, Topological conjugacy of horocycle flows, Amer. J. Math. 105 (1983), 623-632.

17. G. A. Margulis, Non-uniform lattices in semisimple algebraic groups, Lie Groups and Their Representations (I. M. Gelfand, ed.), Wiley, New York, 1975.

18. W. Massey, Algebraic topology: An introduction, Harcourt, Brace, \& World, New York, 1967.

19. M. V. Milovanov, On the extension of automorphisms of uniform discrete subgroups of solvable Lie groups, Dokl. Akad. Nauk BSSR 17 (1973), 892-895.

20. C. C. Moore, Ergodicity of flows on homogeneous spaces, Amer. J. Math. 88 (1966), 154-178.

21. R. Mosak and M. Moskowitz, Analytic density of subgroups of cofinite volume, preprint.

22. G. D. Mostow, Strong rigidity of locally symmetric spaces, Ann. of Math. Studies, no. 78, Princeton Univ. Press, Princeton, N. J., 1973.

23. W. Parry, Metric classification of ergodic nilflows, Amer. J. Math. 93 (1971), 819-829.

24. K. Petersen, Ergodic theory, Cambridge Univ. Press, Cambridge, 1983.

25. G. Prasad, Strong rigidity of Q-rank 1 lattices, Invent. Math. 21 (1973), 255-286.

26. M. S. Raghunathan, Discrete subgroups of Lie groups, Springer-Verlag, New York, 1972.

27. M. Ratner, Rigidity of horocycle flows, Ann. of Math. (2) 115 (1982), 597-614.

28. _ Ergodic theory in hyperbolic space, preprint.

29. M. Saito, Sur certains groupes de Lie resolubles, Sci. Papers of the College of General Education, Univ. of Tokyo, Vol. 7, 1957, pp. 111 and 157-168.

30. V. S. Varadarajan, Lie groups, Lie algebras, and their representations, Springer-Verlag, New York, 1984. 
31. P. Walters, Conjugacy properties of affine transformations of nilmanifolds, Math. Systems Theory $4(1970), 322-326$.

32. D. Witte, Rigidity of some translations on homogeneous spaces, Invent. Math. 81 (1985), 1-27.

33. S. Wolpert, The length spectra as moduli for compact Riemann surfaces, Ann. of Math. (2) 109 (1979), 323-351.

34. R. J. Zimmer, Ergodic theory and semisimple groups, Birkhäuser, Boston, Mass., 1984.

Department of Mathematics, University Of Illinois, URbana, Illinois 61801

Current address: Department of Mathematics, Tufts University, Medford, Massachusetts 02155 